BULLETIN (New Series) OF THE

AMERICAN MATHEMATICAL SOCIETY

Volume 45, Number 4, October 2008, Pages 489-534

S 0273-0979(08)01221-4

Article electronically published on July 2, 2008

\title{
THE IMPORTANCE OF THE SELBERG INTEGRAL
}

\author{
PETER J. FORRESTER AND S. OLE WARNAAR
}

\begin{abstract}
It has been remarked that a fair measure of the impact of Atle Selberg's work is the number of mathematical terms that bear his name. One of these is the Selberg integral, an $n$-dimensional generalization of the Euler beta integral. We trace its sudden rise to prominence, initiated by a question to Selberg from Enrico Bombieri, more than thirty years after its initial publication. In quick succession the Selberg integral was used to prove an outstanding conjecture in random matrix theory and cases of the Macdonald conjectures. It further initiated the study of $q$-analogues, which in turn enriched the Macdonald conjectures. We review these developments and proceed to exhibit the sustained prominence of the Selberg integral as evidenced by its central role in random matrix theory, Calogero-Sutherland quantum many-body systems, Knizhnik-Zamolodchikov equations, and multivariable orthogonal polynomial theory.
\end{abstract}

\section{Contents}

1. Discovery and reappearance 490

1.1. 1941 and $1944 \quad 490$

1.2. The 1950s to the late 1970s - the Mehta integral 491

1.3. More from the 1960s and 1970s - constant term identities 494

1.4. A culmination - the Macdonald conjectures 498

2. Underpinnings of the Selberg integral 501

2.1. The Dixon-Anderson integral 501

2.2. Dotsenko-Fateev integrals 503

2.3. $q$-Integrals and constant terms $\quad 505$

3. The Selberg integral and multivariable orthogonal polynomials $\quad 506$

3.1. Jack polynomial theory

3.2. Classical multivariable orthogonal polynomials 512

4. Recent and current research directions $\quad 514$

4.1. The case of $\gamma$ a positive integer 514

4.2. Random matrix theory 515

4.3. KZ equations and the Mukhin-Varchenko conjecture 519

4.4. Elliptic Selberg integrals 523

4.5. The value distributions of the Riemann $\zeta$ function on the critical line 525

Acknowledgements $\quad 527$

About the authors $\quad 527$

References

Received by the editors March 21, 2008 and, in revised form, April 21, 2008.

2000 Mathematics Subject Classification. Primary 00-02, 33-02.

(C)2008 American Mathematical Society 


\section{Discovery AND REAPPEARANCE}

1.1. 1941 and 1944. With the passing of Atle Selberg on August 6, 2007, at age 90 , it is timely to reflect on his mathematical legacy. Indeed a number of brief articles highlighting some of his most influential mathematical discoveries were written shortly after the news of his death; see e.g., [79]. It is our aim to add to these tributes by giving a more comprehensive account of the mathematics, both pure and applied, related to what now is referred to as the Selberg integral:

$$
\begin{aligned}
S_{n}(\alpha, \beta, \gamma) & :=\int_{0}^{1} \cdots \int_{0}^{1} \prod_{i=1}^{n} t_{i}^{\alpha-1}\left(1-t_{i}\right)^{\beta-1} \prod_{1 \leq i<j \leq n}\left|t_{i}-t_{j}\right|^{2 \gamma} \mathrm{d} t_{1} \cdots \mathrm{d} t_{n} \\
& =\prod_{j=0}^{n-1} \frac{\Gamma(\alpha+j \gamma) \Gamma(\beta+j \gamma) \Gamma(1+(j+1) \gamma)}{\Gamma(\alpha+\beta+(n+j-1) \gamma) \Gamma(1+\gamma)} .
\end{aligned}
$$

The evaluation of this integral is valid for complex parameters $\alpha, \beta, \gamma$ such that (1.2) $\operatorname{Re}(\alpha)>0, \operatorname{Re}(\beta)>0, \operatorname{Re}(\gamma)>-\min \{1 / n, \operatorname{Re}(\alpha) /(n-1), \operatorname{Re}(\beta) /(n-1)\}$, corresponding to the domain of convergence of the integral.

The proof of (1.1) is the subject of Selberg's 1944 paper "Bemerkninger om et multipelt integral" [Remarks on a multiple integral] [145] - the only one of Selberg's works written in Norwegian - published in Norsk Matematisk Tidsskrift. The latter has been compared 23] to the Scandinavian equivalent of the Mathematical Gazette, with contents ranging from short research papers on subjects of general interest to discussions on teaching problems. Selberg himself remarks in his collected works [147] that

This paper was published with some hesitation, and in Norwegian, since I was rather doubtful that the results were new. The journal is one which is read by mathematics-teachers in the gymnasium, and the proof was written out in some detail so it should be understandable to someone who knew a little about analytic functions and analytic continuation.

Selberg's proof of (1.1) proceeds by supposing $\gamma$ is a positive integer, and expanding

$$
\prod_{1 \leq i<j \leq n}\left|t_{i}-t_{j}\right|^{2 \gamma}=\sum_{0 \leq k_{1}, \ldots, k_{n} \leq 2(n-1) \gamma} c_{k_{1}, \ldots, k_{n}} t_{1}^{k_{1}} \cdots t_{n}^{k_{n}} .
$$

Substituting this expansion in the definition of $S_{n}(\alpha, \beta, \gamma)$ allows the resulting integrals to be evaluated by the Euler beta integral [47]

$$
B(\alpha, \beta):=\int_{0}^{1} t^{\alpha-1}(1-t)^{\beta-1} \mathrm{~d} t=\frac{\Gamma(\alpha) \Gamma(\beta)}{\Gamma(\alpha+\beta)},
$$

which itself is (1.1) with $n=1$. The details, in English, of the proof from here on can for example be found in [58, 115]. Perhaps the most significant feature is the final step; it requires analytically continuing $\gamma$ off the integers. Thus with (1.1) established for $\gamma$ a positive integer, the remaining task is to establish its validity for all complex $\gamma$ such that both sides are well defined.

For this purpose Carlson's theorem [27] can be used after noting that both the left- and right-hand side of (1.1) are bounded analytic functions of $\gamma$ for $\operatorname{Re}(\gamma) \geq$ 1 at least. Carlson's theorem applies to functions $f(z)$ analytic for $\operatorname{Re}(z) \geq 0$ satisfying the bound $|f(z)|=\mathrm{O}\left(\mathrm{e}^{\mu|z|}\right), \mu<\pi$. It asserts that if furthermore $f(z)=0$ 
on the nonnegative integers, then, identically, $f(z)=0$. Note that the function $f(z)=\sin \pi z$ shows that the bound $\mu<\pi$ is optimal. Selberg did not make direct use of Carlson's theorem, but rather derived the same result from first principles in the case that $f(z)$ is bounded in the right half-plane. This is all that is required to finalize the proof of (1.1).

Interestingly, although 145] contains the first proof of (1.1), it is not the first time it appeared in print. This occurred three years earlier (albeit with the change of variables $t_{i}=s_{i} /\left(1+s_{i}\right)$ so that $\left.s_{i} \in[0, \infty)\right)$ in Selberg's 1941 paper "Über einen Satz von A. Gelfond" (On a theorem of A. Gelfond) [144]. Like [145], this earlier paper appeared in a Norwegian journal, this time Archiv for Mathematik og Naturvidenskab, known for having Sophus Lie as one of its founders. In a footnote Selberg remarks

Leider habe ich die Formel (11) [The Selberg integral] nirgends in der Litteratur finden können, ein Beweis hier zu bringen scheint aber nicht angebracht, da die Arbeit sonst zu sehr anschwellen würde; sollte sich aber herausstellen, dass die Formel neu wäre, beabsichtige ich später ein Beweis zu veröffentlichen.

[Unfortunately I have been unable to find formula (11) [The Selberg integral] in the literature. To present a proof here, however, seems inappropriate, as it would make this paper significantly longer. If it turns out that the formula is new, I intend to publish a proof at a later date.]

Curiously, Selberg used his integral in 144] to prove a result of some similarity to Carlson's theorem. As already noted, the latter is itself an ingredient in Selberg's proof of (1.1). Selberg's result relates to entire functions $f(z)$ of exponential type $\sigma(f)$, defined by

$$
\sigma(f):=\limsup _{r \rightarrow \infty} \frac{1}{r} \log \left(\max _{|z|=r}|f(z)|\right) .
$$

A theorem of Hardy and Pólya 22, 132] states that $f$ is a polynomial if it takes integer values at the nonnegative integers, and $\sigma(f)<\log 2$. The transcendental function $f(z)=2^{z}$ shows that this bound is optimal. A. Gelfond 68 generalized this by proving that $f$ is a polynomial if it and its first $n-1$ derivatives take integer values at the nonnegative integers, and if $\sigma(f)<n \log (1+\exp (1 / n-1))$. However, for $n>1$ this bound is not optimal. By using his integral, Selberg improved Gelfond's bound for $n>1$ to $\sigma(f)<\log m_{n}$, where $m_{n}$ is the minimum value of $\prod_{i=1}^{n}\left(1+y_{i}\right)$ under the conditions $y_{i}>0, y_{1} \cdots y_{n}=\exp (1-n)$ and $\prod_{1 \leq i<j \leq n}\left|y_{i}^{-1}-y_{j}^{-1}\right|=1$. This improves Gelfond's result since

$$
\prod_{i=1}^{n}\left(1+y_{i}\right)>\left(1+\mathrm{e}^{1 / n-1}\right)^{n} .
$$

1.2. The $1950 \mathrm{~s}$ to the late $1970 \mathrm{~s}$ - the Mehta integral. For over thirty years the Selberg integral went essentially unnoticed. It was used only once -in the special case $\alpha=\beta=1, \gamma=2$-in a study by S. Karlin and L.S. Shapley relating to the volume of a certain moment space, published in 1953 [92].

Around ten years later, there was again good reason to make use of (1.1). Building upon the earlier work of E.P. Wigner in the 1950s, F.J. Dyson wrote a series of papers on the statistical theory of energy levels of complex systems. These papers 
ranged from the theory's foundations to its practical use in the analysis of experimental data. This last topic was addressed in Part V of the series [116], in a paper, written jointly with M.L. Mehta and published in 1963, which also summarizes both the status of the theory and open problems from that date.

A basic point is that random Hermitian matrices are used to model the highly excited states of complex nuclei. These matrices are taken to have real, complex or real quaternion elements, and they correspond to the quantum system having time reversal symmetry, no time reversal symmetry, or time reversal symmetry with an odd number of spin $1 / 2$ particles, respectively.

The random Hermitian matrices $H$ can be constructed from Gaussian matrices with independent elements according to $H=\frac{1}{2}\left(X+\bar{X}^{T}\right)$. The elements of $X$ are standard normals $\mathrm{N}[0,1]$ in the real case, while in the complex and real quaternion cases they are chosen independently from $\mathrm{N}[0,1]+\mathrm{i} \mathrm{N}[0,1]$. We note that our choice of variance is nonstandard; more conventionally, the real parts of entries are chosen from $\mathrm{N}[0,1 / \sqrt{\beta}]$ with $\beta=1,2$ and 4 in the real, complex and real quaternion case, respectively. Real quaternion elements are themselves $2 \times 2$ blocks of the form

$$
\left[\begin{array}{cc}
z & w \\
-\bar{w} & \bar{z}
\end{array}\right]
$$

and so contain only two independent complex numbers. In general the eigenvalues of Hermitian matrices with real quaternion elements are doubly degenerate. The above-mentioned ensembles of random matrices are referred to as the Gaussian orthogonal, unitary and symplectic ensembles (abbreviated GOE, GUE, GSE), respectively.

For each of the three Gaussian ensembles, the joint probability density function (PDF) for the eigenvalues can be computed explicitly as [115]

$$
\frac{1}{(2 \pi)^{n / 2} F_{n}(\beta / 2)} \prod_{i=1}^{n} \mathrm{e}^{-t_{i}^{2} / 2} \prod_{1 \leq i<j \leq n}\left|t_{i}-t_{j}\right|^{\beta} .
$$

Here $\beta=1,2,4$ for the GOE, GUE and GSE, respectively, while $F_{n}$ is the normalization

$$
F_{n}(\gamma):=\frac{1}{(2 \pi)^{n / 2}} \int_{-\infty}^{\infty} \cdots \int_{-\infty}^{\infty} \prod_{i=1}^{n} \mathrm{e}^{-t_{i}^{2} / 2} \prod_{1 \leq i<j \leq n}\left|t_{i}-t_{j}\right|^{2 \gamma} \mathrm{d} t_{1} \cdots \mathrm{d} t_{n}
$$

referred to as Mehta's integral. In [116] Mehta and Dyson evaluated $F_{n}(\beta / 2)$ for each of the the three special values of $\beta$. Combining this with the evaluations for $n=2$ and $n=3$ for general $\beta$, led them to conjecture that

$$
F_{n}(\gamma)=\prod_{j=1}^{n} \frac{\Gamma(1+j \gamma)}{\Gamma(1+\gamma)}
$$


Assuming the validity of (1.6) for general $\gamma$, certain averages associated with (1.4) at the special random matrix couplings $\beta=1,2,4$ are accessible. This becomes apparent by writing (1.4) in the form

$$
\frac{\mathrm{e}^{-\beta U}}{(2 \pi)^{n / 2} F_{n}(\beta / 2)}
$$

where

$$
U=\frac{1}{2 \beta} \sum_{i=1}^{n} t_{i}^{2}-\sum_{1 \leq i<j \leq n} \log \left|t_{i}-t_{j}\right| .
$$

The mean $\langle U\rangle$ for a given $\beta$ is now computed by taking the logarithmic derivative of the normalization $F_{n}(\beta / 2)$. A further differentiation with respect to $\beta$ then yields the fluctuation $\left\langle U^{2}\right\rangle-\langle U\rangle^{2}$.

The form (1.7) highlights an analogy with the equilibrium statistical mechanics of a classical gas of $n$ particles on the line, at inverse temperature $\beta$, interacting via a repulsive logarithmic Coulomb potential and confined by a harmonic well. The quantity $\exp (-\beta U)$ is referred to as the Boltzmann factor. This interpretation plays a prominent role in Dyson's series of works. Indeed, the notation for the averages used above stems from the statistical physics literature (and corresponds to the mean energy and specific heat of the Coulomb gas) and may be substituted by the mean $\mu(U)$ and variance $\sigma^{2}(U)$, respectively. In such an interpretation, one would typically scale $t_{i} \mapsto \sqrt{\beta} t_{i}$ in (1.4) so that $U$ in (1.7) is independent of $\beta$ (formally set $\beta=1$ therein). A natural question then is to seek the configuration of $\left\{t_{i}\right\}$ giving the minimum of $U$. As noted in [116], a result of T.J. Stieltjes (see, e.g., [6]) gives that the minimum occurs at the zeros of the Hermite polynomial $H_{n}(x)$.

It is not hard to see that the Selberg integral can be used to evaluate Mehta's integral thus proving the conjecture (1.6). By the change of variables $t_{i} \mapsto$ $\left(1-t_{i} / L\right) / 2$ in (1.1)

$$
\lim _{L \rightarrow \infty} 2^{L^{2}}(2 L)^{n+n(n-1) \gamma} S_{n}\left(L^{2} / 2, L^{2} / 2, \gamma\right)=F_{n}(\gamma) .
$$

Use of Stirling's formula to compute the same limit on the right-hand side of (1.1) then gives (1.6). However, in 1963 when Mehta and Dyson published their conjecture, the Selberg integral was essentially unknown and so this method of proof was not available to them.

The Mehta-Dyson conjecture received more prominence with its appearance in the first edition of Mehta's book Random Matrices and the Statistical Theory of Energy Levels, published in 1967 [113]. In 1974 Mehta submitted the conjecture to the problems section of SIAM Review [114], thus gaining exposure to an even wider mathematical audience. A proof, exactly the one mentioned in the previous paragraphs, was finally uncovered in the late 1970s by Selberg's IAS colleague Enrico Bombieri. The remarkable story behind this proof is best told in Bombieri's own words 24]:

Since 1976 I had been studying elementary methods in prime number theory and in particular a several variable extension of Chebyshev's well-known method to obtain upper and lower bounds for the number of primes up to a given bound. In the course of my researches I came across the problem of the asymptotic computation 
of certain multiple integrals, the simplest being

$$
\int \prod_{i=1}^{n} z_{i}^{-r-1}\left(1-z_{i}\right)^{p} \prod_{\substack{i, j=1 \\ i \neq j}}^{n}\left(z_{i}-z_{j}\right)^{q} \mathrm{~d} z_{1} \cdots \mathrm{d} z_{n}
$$

where $p, q, r$ are large positive integers and the integral is extended to the product of the unit circles $\left|z_{i}\right|=1$ or to $[0,1]^{n}$.

The integral is related to a partition function for the one-dimensional Coulomb gas on the unit circle $|z|=1$ with a fixed point charge at $z=1$, as it was explained to me by my friend and colleague Tom Spencer, so I went to Dyson and asked him whether physicists had encountered such things before; maybe he could save me some efforts.

Dyson told me that for $q=1$ and 2 an integral of this type, over the real line with a gaussian measure, had indeed been studied and he referred me to a book by Mehta. Then I went to see Atle to ask his opinion about what I was doing in order to study the distribution of primes and whether he felt it was of any interest and whether he had any opinion on it.

He immediately recognized my integral as a complex version of the generalized beta integral he had studied before and he gave me an off-print of his paper. It was not difficult to follow his proof, given for an integral over $[0,1]^{n}$, and use a classical method to write a Beta integral as a complex integral to solve my problem of computing my integral exactly. The multiple integral over $[0,1]^{n}$ is of course Selberg's integral, as in that case arithmetical applications require $r$ to be a large negative (not positive) integer. It was also quite easy to get a confluent form of the Selberg integral and compute exactly the Mehta integrals for a general value of the parameter and make physicists happy.

Since this was of interest to Dyson, I went back to Dyson and told him that using the Selberg integral one could compute the integral of interest to physicists.

1.3. More from the $1960 \mathrm{~s}$ and $1970 \mathrm{~s}$ - constant term identities. The consideration of time reversal symmetry leading to three ensembles of Hermitian matrices applies equally well to unitary matrices [45]. A conventional time reversal symmetry requires that $U=U^{T}$, no time reversal symmetry imposes no constraint, whilst a time reversal symmetry for a system with an odd number of spin 1/2 particles requires $U=U^{D}$ (here $D$ denotes the quaternion dual; see, e.g., [58, Ch. 2]). Choosing such matrices with a uniform probability then gives what are referred to as the circular orthogonal, unitary and symplectic ensembles (COE, CUE and CSE), respectively. Their joint eigenvalue PDFs are given explicitly by

$$
\frac{1}{(2 \pi)^{n} C_{n}(\beta / 2)} \prod_{1 \leq i<j \leq n}\left|\mathrm{e}^{\mathrm{i} \theta_{i}}-\mathrm{e}^{\mathrm{i} \theta_{j}}\right|^{\beta}
$$


where $C_{n}$ is the normalization

$$
C_{n}(\gamma)=\frac{1}{(2 \pi)^{n}} \int_{-\pi}^{\pi} \cdots \int_{-\pi}^{\pi} \prod_{1 \leq i<j \leq n}\left|\mathrm{e}^{\mathrm{i} \theta_{i}}-\mathrm{e}^{\mathrm{i} \theta_{j}}\right|^{2 \gamma} \mathrm{d} \theta_{1} \cdots \mathrm{d} \theta_{n}
$$

and $\beta=1,2,4$ for the COE, CUE and CSE, respectively.

As for (1.5), the random matrix calculations give (1.10) in terms of gamma functions for the three special values of $\beta$. Furthermore, the case $n=2$ for general $\beta$ can be related to the Euler beta integral (1.3), whilst the case $n=3$ gives a sum which is a special instance of an identity of Dixon for a well-poised ${ }_{3} F_{2}$ series [6],

$$
\begin{aligned}
{ }_{3} F_{2}\left(\begin{array}{c}
a, b, c \\
1+a-b, 1+
\end{array}\right. & a-c ; 1) \\
& =\frac{\Gamma\left(1+\frac{a}{2}\right) \Gamma(1+a-b) \Gamma(1+a-c) \Gamma\left(1+\frac{a}{2}-b-c\right)}{\Gamma(1+a) \Gamma\left(1+\frac{a}{2}-b\right) \Gamma\left(1+\frac{a}{2}-c\right) \Gamma(1+a-b-c)} .
\end{aligned}
$$

Based on all of these results, Dyson [45], in Part I of his series of papers, made the conjecture

$$
C_{n}(\gamma)=\frac{\Gamma(1+n \gamma)}{\Gamma^{n}(1+\gamma)}
$$

In the same paper, Dyson observed that with $\gamma$ a nonnegative integer, say $k, 1.10$ can be rewritten as the constant term (CT) in a Laurent expansion. This allows (1.12) to be rewritten as

$$
\mathrm{CT} \prod_{1 \leq i<j \leq n}\left(1-\frac{x_{i}}{x_{j}}\right)^{k}\left(1-\frac{x_{j}}{x_{i}}\right)^{k}=\frac{(k n) !}{(k !)^{n}}
$$

This constant term identity, and thus, by Carlson's theorem, the conjecture (1.12), was soon proved by J. Gunson and K. Wilson [171], and later in a very efficient analysis by I.J. Good [69]. Gunson's proof is mentioned in [45], but is not published; reference in is often made to [70] although it actually contains the proof of another conjecture of Dyson. Twenty years after his proof, Wilson was to receive the Nobel Prize in physics for his work on the renormalisation group approach to the study of critical phenomena; 171] is his first publication.

In their proofs, Wilson and Good both took advantage of the extra degrees of freedom offered by Dyson's more general conjecture

$$
\mathrm{CT} \prod_{\substack{i, j=1 \\ i \neq j}}^{n}\left(1-\frac{x_{i}}{x_{j}}\right)^{a_{i}}=\frac{\left(a_{1}+\cdots+a_{n}\right) !}{a_{1} ! \cdots a_{n} !}
$$

also contained in [45]. The formulation of (1.14) was in turn motivated by the extra degrees of freedom permitted by Dixon's identity (1.11), to which it reduces when $n=3$.

In fact, as observed by R. Askey [11], the Selberg integral can be used to prove Dyson's conjecture (1.12) directly without the need for (1.14). Askey's observation 
is based on the easily established general identity

$$
\begin{aligned}
& \int_{0}^{1} \cdots \int_{0}^{1}\left(t_{1} \cdots t_{n}\right)^{\zeta-1} f\left(t_{1}, \ldots, t_{n}\right) \mathrm{d} t_{1} \cdots \mathrm{d} t_{n} \\
& =\left(\frac{1}{2 \sin \pi \zeta}\right)^{n} \int_{-\pi}^{\pi} \cdots \int_{-\pi}^{\pi} \mathrm{e}^{\mathrm{i} \zeta\left(\theta_{1}+\cdots+\theta_{n}\right)} f\left(-\mathrm{e}^{\mathrm{i} \theta_{1}}, \ldots,-\mathrm{e}^{\mathrm{i} \theta_{n}}\right) \mathrm{d} \theta_{1} \cdots \mathrm{d} \theta_{n},
\end{aligned}
$$

valid for $f$ a Laurent polynomial and $\operatorname{Re}(\zeta)$ large enough so that the left-hand side exists. Applying (1.15) to the Selberg integral with $\beta$ a positive integer and $\gamma$ a nonnegative integer, shows that

$$
S_{n}(\alpha, \beta, \gamma)=(-1)^{n+\left(\begin{array}{c}
n \\
2
\end{array}\right) \gamma}\left(\frac{\pi}{\sin \pi b}\right)^{n} M_{n}(a, b, \gamma),
$$

where $\alpha:=-b-(n-1) \gamma, \beta:=a+b+1$ and

$$
\begin{aligned}
M_{n}(a, b, \gamma):=\frac{1}{(2 \pi)^{n}} \int_{-\pi}^{\pi} \cdots \int_{-\pi}^{\pi} \prod_{i=1}^{n} \mathrm{e}^{\frac{1}{2} \mathrm{i} \theta_{i}(a-b)}\left|1+\mathrm{e}^{\mathrm{i} \theta_{i}}\right|^{a+b} \\
\quad \times \prod_{1 \leq i<j \leq n}\left|\mathrm{e}^{\mathrm{i} \theta_{i}}-\mathrm{e}^{\mathrm{i} \theta_{j}}\right|^{2 \gamma} \mathrm{d} \theta_{1} \cdots \mathrm{d} \theta_{n} .
\end{aligned}
$$

From (1.16), the Selberg integral, the reflection formula and finally Carlson's theorem, it follows that

$$
M_{n}(a, b, \gamma)=\prod_{j=0}^{n-1} \frac{\Gamma(1+a+b+j \gamma) \Gamma(1+(j+1) \gamma)}{\Gamma(1+a+j \gamma) \Gamma(1+b+j \gamma) \Gamma(1+\gamma)},
$$

for $a, b, \gamma \in \mathbb{C}$ such that

$$
\operatorname{Re}(a+b+1)>0, \operatorname{Re}(\gamma)>-\min \{1 / n, \operatorname{Re}(a+b+1) /(n-1)\} .
$$

For $a=b=0$ this is Dyson's conjecture (1.12).

The change of variables $\mathrm{e}^{\mathrm{i} \theta_{i}}=\left(\mathrm{i}-t_{i}\right) /\left(\mathrm{i}+t_{i}\right)$ maps the unit circle onto the real line via a stereographic projection. Applying this to the integral (1.17) leads to

$$
\begin{aligned}
& \frac{1}{(2 \pi)^{n}} \int_{-\infty}^{\infty} \cdots \int_{-\infty}^{\infty} \prod_{i=1}^{n} \frac{1}{\left(1+\mathrm{i} t_{i}\right)^{\alpha}\left(1-\mathrm{i} t_{i}\right)^{\beta}} \prod_{1 \leq i<j \leq n}\left|t_{i}-t_{j}\right|^{2 \gamma} \mathrm{d} t_{1} \cdots \mathrm{d} t_{n} \\
& =2^{-n(\alpha+\beta-1)+n(n-1) \gamma} \prod_{j=0}^{n-1} \frac{\Gamma(\alpha+\beta-1-(n+j-1) \gamma) \Gamma(1+(j+1) \gamma)}{\Gamma(\alpha-j \gamma) \Gamma(\beta-j \gamma) \Gamma(1+\gamma)} .
\end{aligned}
$$

When $n=1$, this is simply the Cauchy beta integral.

In the letter to Dyson reprinted on the next page, Selberg communicated the multiple Cauchy integral (1.19). Subsequently, in a letter to Askey dated 25 March 1980, he mentioned both (1.17) and (1.19), and pointed out their exact relationship. The first time (1.17) appeared in print was in W.G. Morris' $1982 \mathrm{PhD}$ thesis [122]. In his thesis Morris provided a proof of (1.17) along the lines of Selberg's proof of (1.1) and applied it to obtain constant term identities. For these reasons (1.17) is now commonly referred to as the Morris integral. 
THE IMPORTANCE OF THE SELBERG INTEGRAL

497

THE INSTITUTE FOR ADVANCED STUDY

PRINCETON, NEW JERSEY 08540

SCHOOL OF MATHEMATICS

Dear Freedman,

$2 / 27-79$

Thanks for your work. Actually 0 found the formula in 1941. I had mot really planned to publish anything about if, lent was later soled to contribute an artie in norwegian to this formal and thought his might be suitable for that audience.

Of corse, I did have a bit mure along the ne limes, but did mot evishto make the oulvele to bulky. Obvious an limiting cases like the analog of Eulas integral for the $P$. function

(1) $\int_{0}^{\infty} \cdots \int_{0}^{\infty}\left(t_{1} \ldots t_{m}\right)^{x-1} e^{-\left(t_{1}+\cdots t_{n}\right)}(\Delta(t))^{2 z} d t_{1} \ldots d t_{n}$, which can be derived in a Similar way as the other limiting case yon mention.

Another related (hough mort so obviously) formula is

(2)

$$
\begin{aligned}
& \text { formula in } \int_{-\infty}^{\infty} \cdots \int_{-\infty}^{\infty} \frac{|\Delta(t)|^{2 z}}{\prod_{j=1}^{n}\left(1+i \bar{y}_{j}\right)^{x}\left(1-i t_{j}\right)^{y}} d t_{1} . . d t_{n}= \\
& =(4 \pi)^{n}-m(x+y-(m-1) z) \prod_{\nu=1}^{n} \frac{\Gamma(1+v z) \Gamma(x+y-1-(m+\nu-2) z)}{\Gamma(1+z) \Gamma(x-(\nu-1) z) \Gamma(y-(v-1) z}
\end{aligned}
$$

This is rabid for complex $x, y, z$ for which the integral inverses absolutely (Conditions easy to find a bot tedious to unite down). From (2) one can cover (using if for the eave $x=y$ ) again obtain the formal you refer to in your letter as a limiting case.

O had cut thought about these Sining s now for more than thirty years when Boobies consulted me about a problem of his which if seemed to me coed be handled bey roving ny frnmila from the 1944 paper. That here had been come interest in analryons. integrals among pleqpicials was completely silicons bo me curtis sins.

Gems siree?

ate 
1.4. A culmination - the Macdonald conjectures. In 1982 I.G. Macdonald [108] published his now famous ex-conjectures, generalizing the Mehta integral (1.5) to all finite reflection or Coxeter groups $G$, and the Dyson constant term identity (1.13) to all finite root systems.

Let $G$ be a finite group of isometries of $\mathbb{R}^{n}$, generated by reflections in $N$ hyperplanes. Normalize (up to sign) so that the equations for the hyperplanes take the form

$$
a_{i 1} x_{1}+\cdots+a_{i n} x_{n}=0 \quad \text { with } \quad a_{i 1}^{2}+\cdots+a_{i n}^{2}=2,
$$

where $i$ labels the hyperplanes, and form the polynomial

$$
P(x)=\prod_{i=1}^{N}\left(a_{i 1} x_{1}+\cdots+a_{i n} x_{n}\right) .
$$

Geometrically, $2^{-N / 2} P(x)$ gives the product of the distances of the point $x \in \mathbb{R}$ to the $N$ hyperplanes.

By its action on $\mathbb{R}^{n}$, the group $G$ acts on polynomials in $x=\left(x_{1}, \ldots, x_{n}\right)$. The polynomials that are invariant under the action of $G$ are referred to as $G$-invariant polynomials. They form an $\mathbb{R}$-algebra $\mathbb{R}\left[f_{1}, \ldots, f_{n}\right]$ generated by $n$ algebraically independent polynomials $f_{1}, \ldots, f_{n}$ of degrees $d_{1}, \ldots, d_{n}$. Unlike the set of $f_{i}$ 's, the set of $d_{i}$ 's is uniquely determined by the underlying reflection group.

A final ingredient required in the Macdonald conjectures is the Gaussian measure $\varphi$ on $\mathbb{R}^{n}$

where $|x|^{2}:=\sum_{i=1}^{n} x_{i}^{2}$.

$$
\mathrm{d} \varphi(x):=\frac{\mathrm{e}^{-|x|^{2} / 2}}{(2 \pi)^{n / 2}} \mathrm{~d} x_{1} \cdots \mathrm{d} x_{n},
$$

With the above notation, Macdonald's (ex)-conjecture [108, Conjecture 5.1] states that for each finite reflection group $G$

$$
\int_{\mathbb{R}^{n}}|P(x)|^{2 \gamma} \mathrm{d} \varphi(x)=\prod_{i=1}^{n} \frac{\Gamma\left(1+d_{i} \gamma\right)}{\Gamma(1+\gamma)} .
$$

For the three infinite families of crystallographic reflection groups (or reflection groups of Weyl type) $\mathrm{A}_{n-1}, \mathrm{~B}_{n}$ and $\mathrm{D}_{n}$, the Macdonald conjecture follows as a limit of the Selberg integral. For type $\mathrm{A}_{n-1}$ this corresponds to Bombieri's proof of the Mehta integral mentioned earlier. For types $\mathrm{B}_{n}$ and $\mathrm{D}_{n}$ this is due to $\mathrm{A}$. Regev, although the actual proof appeared for the first time in the paper by Macdonald, to whom Regev communicated his results.

Around the same time as Macdonald formulated his conjectures, Regev was studying the large $n$ behaviour of sums of the form $S_{\ell}^{\beta}(n):=\sum_{\lambda}\left(d_{\lambda}\right)^{\beta}$, where the sum is over partitions $\lambda$ of at most $\ell$ parts, and $d_{\lambda}$ is the dimension of the irreducible $\mathfrak{S}_{n}$ character $[\lambda]$. Combining the hook-length formula for $d_{\lambda}$ with a careful asymptotic analysis, Regev showed [138] (see also [32]) that the asymptotics of sums like $S_{\ell}^{\beta}(n)$ leads exactly to Mehta's integral. Regev remarks [139]

From reactions to preprints and talks on [138], first from Richard Stanley (who in 1978 attended my seminar talk at UCSD) then from Freeman Dyson, I learned about the Mehta and the Macdonald conjectures. In a letter, Dyson also mentioned that the Mehta conjecture had just been verified - by applying the Selberg integral. William Beckner then showed me the details of how to deduce the 
Mehta - and some other integrals - from the Selberg integral. I worked on the other classical cases of the Macdonald conjecture and managed to verify these shortly afterwards, in 1979.

The Coxeter group $\mathrm{A}_{n-1}$ is the symmetry group of the $(n-1)$-simplex. It is a group of order $n$ ! generated by the $n(n-1) / 2$ hyperplanes

$$
x_{i}-x_{j}=0 \text { for } 1 \leq i<j \leq n,
$$

and is isomorphic to the symmetric group $\mathfrak{S}_{n}$. All of the ingredients in (1.20) can thus easily be determined explicitly. The polynomial $P(x)$ is given by the Vandermonde product

$$
P(x)=\prod_{1 \leq i<j \leq n}\left(x_{i}-x_{j}\right)=: \Delta(x),
$$

and the $G$-invariant polynomials are given by the symmetric polynomials in $x$. One of the classical bases for the algebra of symmetric functions is given by the elementary symmetric functions $\left\{e_{1}, \ldots, e_{n}\right\}$ with

$$
e_{r}(x)=\sum_{1 \leq i_{1}<i_{2}<\cdots<i_{r} \leq n} x_{i_{1}} x_{i_{2}} \cdots x_{i_{r}} .
$$

Accordingly, the set of degrees $d_{i}$ is given by $\{1,2, \ldots, n\}$, and (1.20) reduces to Mehta's integral (1.5).

The Coxeter groups $\mathrm{B}_{n}$ and $\mathrm{D}_{n}$ are the symmetry groups of the $n$-cube and $n$-demicube, and for these groups (1.20) takes the form

$$
\int_{\mathbb{R}^{n}} \prod_{i=1}^{n}\left(2\left|x_{i}\right|^{2}\right)^{\gamma} \prod_{1 \leq i<j \leq n}\left|x_{i}^{2}-x_{j}^{2}\right|^{2 \gamma} \mathrm{d} \varphi(x)=\prod_{i=1}^{n} \frac{\Gamma(1+2 i \gamma)}{\Gamma(1+\gamma)},
$$

and

$$
\int_{\mathbb{R}^{n}} \prod_{1 \leq i<j \leq n}\left|x_{i}^{2}-x_{j}^{2}\right|^{2 \gamma} \mathrm{d} \varphi(x)=\frac{\Gamma(1+n \gamma)}{\Gamma(1+\gamma)} \prod_{i=1}^{n-1} \frac{\Gamma(1+2 i \gamma)}{\Gamma(1+\gamma)},
$$

respectively. Making the changes $t_{i}=x_{i}^{2} /(2 L), \alpha=c+1 / 2$ and $\beta=L+1$ in the Selberg integral and letting $L$ tend to infinity gives

$$
\int_{\mathbb{R}^{n}} \prod_{i=1}^{n}\left(2\left|x_{i}\right|^{2}\right)^{c} \prod_{1 \leq i<j \leq n}\left|x_{i}^{2}-x_{j}^{2}\right|^{2 \gamma} \mathrm{d} \varphi(x)=\prod_{j=0}^{n-1} \frac{\Gamma(1+2 c+2 j \gamma) \Gamma(1+(j+1) \gamma)}{\Gamma(1+c+j \gamma) \Gamma(1+\gamma)},
$$

where use has been made of Legendre's duplication formula. The above integral, known as the $\mathrm{BC}_{n}$ Mehta integral, leads to the $\mathrm{B}_{n}$ and $\mathrm{D}_{n}$ integrals by setting $c=\gamma$ and $c=0$, respectively.

In his original paper, Macdonald established several other instances of his conjecture, not relying on the Selberg integral. For $\gamma=1$, Macdonald presented a uniform proof for all crystallographic reflection groups. Another case of (1.20) one that may be verified by purely elementary means - is that of the dihedral group $\mathrm{I}_{2}(m)$, the symmetry group of a regular $m$-gon.

A uniform proof of Macdonald's conjecture for all crystallographic reflection groups- $\mathrm{A}_{n-1}, \mathrm{~B}_{n}, \mathrm{D}_{n}, \mathrm{E}_{6}, \mathrm{E}_{7}, \mathrm{E}_{8}, \mathrm{~F}_{4}$ and $\mathrm{G}_{2}$-was found in 1989 by E. Opdam [129] using the Heckman-Opdam theory of hypergeometric shift operators 76, 129]. Several years later, combined theoretical and computer efforts by Opdam [130] and 
F. Garvan [63, 64] also dealt with the remaining noncrystallographic groups $\mathrm{H}_{3}$ and $\mathrm{H}_{4}$, thereby settling Macdonald's conjecture in full.

In his paper Macdonald put forward many further conjectures related to root systems. One of these [108, Conjecture 2.7] is the generalization of Dyson's constant term identity (1.13) to arbitrary (finite) root systems. Let $\Phi$ be a root system (not necessarily reduced) with corresponding Weyl group $\mathcal{W}$. For $\alpha \in \Phi$ let $\exp (\alpha)$ be a formal exponential. Denote the degrees of the fundamental invariants of $\mathcal{W}$ by $d_{1}, \ldots, d_{l}$. The $d_{i}$ may, for example, be obtained from the simple formula

$$
\prod_{\alpha \in \Phi^{+}} \frac{1-t^{\mathrm{ht}(\alpha)+s(\alpha)}}{1-t^{\mathrm{ht}(\alpha)}}=\prod_{i=1}^{l} \frac{1-t^{d_{i}}}{1-t}
$$

where $\Phi^{+}$is the set of positive roots of the root system, $\operatorname{ht}(\alpha)$ is the height of the root $\alpha$ and $s(\alpha)=1$ if $\alpha / 2 \notin \Phi^{+}$and $s(\alpha)=2$ if $\alpha / 2 \in \Phi^{+}$; the case $s(\alpha)=2$ can only occur for nonreduced root systems. Then Macdonald's constant term conjecture asserts that

$$
\mathrm{CT} \prod_{\alpha \in \Phi}\left(1-\mathrm{e}^{\alpha}\right)^{k}=\prod_{i=1}^{l}\left(\begin{array}{c}
d_{i} k \\
k
\end{array}\right) .
$$

For the root system $\mathrm{A}_{n-1}, \Phi=\left\{\epsilon_{i}-\epsilon_{j} \mid 1 \leq i, j \leq n, i \neq j\right\}$, where $\epsilon_{i}$ the $i$ th standard unit vector in $\mathbb{R}^{n}$. The degrees in this case are given by $2,3, \ldots, n$ so that, after the identification $\exp \left(\epsilon_{i}-\epsilon_{j}\right)=x_{i} / x_{j}$, one recovers Dyson's conjecture.

When $k=1$, equation (1.22) simply follows from the classical Weyl denominator formula. Macdonald also proved the $k=2$ case using algebraic techniques. Once again the Selberg integral implies the conjecture for the infinite series $\mathrm{B}_{n}, \mathrm{C}_{n}, \mathrm{D}_{n}$ and $\mathrm{BC}_{n}$. Since the first three are all contained in the last, the most succinct derivation arises by slightly generalizing the problem-Macdonald does this for all root systems in [108, Conjecture 2.3] - and considering the constant term of

$$
\mathrm{CT} \prod_{\alpha \in \Phi_{\mathrm{BC}_{n}}}\left(1-\mathrm{e}^{\alpha}\right)^{k_{\alpha}} .
$$

Here

$$
\Phi_{\mathrm{BC}_{n}}=\left\{ \pm \epsilon_{i} \mid 1 \leq i \leq n\right\} \cup\left\{ \pm 2 \epsilon_{i} \mid 1 \leq i \leq n\right\} \cup\left\{ \pm \epsilon_{i} \pm \epsilon_{j} \mid 1 \leq i<j \leq n\right\}
$$

is the $\mathrm{BC}_{n}$ root system and $k_{\alpha}=k_{1}$ if $\alpha$ is of type $\pm \epsilon_{i}, k_{\alpha}=k_{3}$ if $\alpha$ is of type $\pm 2 \epsilon_{i}$ and $k_{\alpha}=k_{2}$ otherwise. The root systems $\mathrm{B}_{n}, \mathrm{C}_{n}$ and $\mathrm{D}_{n}$ are then obtained by taking $k_{3}=0$ or $k_{1}=0$ or $k_{1}=k_{3}=0$, respectively. By the substitution $\exp \left(\epsilon_{i}\right) \mapsto \exp \left(2 \mathrm{i} \theta_{i}\right)$, it follows that

$$
\begin{aligned}
& \text { 23) } \prod_{\alpha \in \Phi_{\mathrm{BC} n}}\left(1-\mathrm{e}^{\alpha}\right)^{k_{\alpha}}=\frac{2^{n(a+b)+n(n-1) c}}{\pi^{n}} \\
& \times \int_{0}^{\pi} \cdots \int_{0}^{\pi} \prod_{i=1}^{n} \sin ^{a}\left(\theta_{i}\right) \cos ^{b}\left(\theta_{i}\right) \prod_{1 \leq i<j \leq n} \sin ^{c}\left(\theta_{i}-\theta_{j}\right) \sin ^{c}\left(\theta_{i}+\theta_{j}\right) \mathrm{d} \theta_{1} \cdots \mathrm{d} \theta_{n},
\end{aligned}
$$

with $a=2 k_{1}+2 k_{3}, b=2 k_{3}$ and $c=2 k_{2}$. Introducing new integration variables $t_{i}=\sin ^{2}\left(\theta_{i}\right)$ for all $1 \leq i \leq n$, the integral on the right transforms into the Selberg 
integral. Hence, by (1.1) and the Legendre duplication formula,

$$
\text { CT } \begin{aligned}
\prod_{\alpha \in \Phi_{\mathrm{BC} n}} & \left(1-\mathrm{e}^{\alpha}\right)^{k_{\alpha}} \\
& =\frac{4^{n\left(k_{1}+2 k_{3}\right)+n(n-1) k_{2}}}{\pi^{n}} S_{n}\left(k_{1}+k_{3}+\frac{1}{2}, k_{3}+\frac{1}{2}, k_{2}\right) \\
& =\prod_{i=0}^{n-1} \frac{\left(k_{2}+i k_{2}\right) !\left(2 k_{1}+2 k_{3}+2 i k_{2}\right) !\left(2 k_{3}+2 i k_{2}\right) !}{k_{2} !\left(k_{1}+k_{3}+i k_{2}\right) !\left(k_{3}+i k_{2}\right) !\left(k_{1}+2 k_{3}+(n+i-1) k_{2}\right) !} .
\end{aligned}
$$

In a not dissimilar manner, D. Zeilberger 175] showed that the $n=3$ case of the Morris integral (1.17) leads to the Macdonald conjecture for the exceptional root system $\mathrm{G}_{2}$. This result later found application in a study linking random matrix theory to number theoretical $L$-functions [97], see also Section 4.5.

A unified proof of (1.22) for all root systems, based on hypergeometric shift operators, is again due to Opdam [129]. Pages 508-509 below contain an outline of this proof for the root system $\mathrm{A}_{n-1}$.

\section{Underpinnings of THE SElBerg InTEgral}

2.1. The Dixon-Anderson integral. The Euler beta integral (1.3) has for its integrand the product of power functions $x^{\alpha-1} y^{\beta-1}$ with $y=1-x$. It is evaluated as a ratio of gamma functions, which in turn are integrals over the product of a power function and an exponential function. Now let $\mathbb{F}_{q}$ be a finite field of $q$ elements, where $q=p^{n}$ with $p$ an odd prime and $n$ a positive integer. Then the role of power and exponential functions is played by multiplicative and additive characters $\tau: \mathbb{F}_{q}^{*} \rightarrow \mathbb{C}^{*}$ and $\psi: \mathbb{F}_{q} \rightarrow \mathbb{C}^{*}$. These characters can be used to define the finite field analogues of the gamma and beta functions, known as the Gauss and Jacobi sums, in such a way that a finite field analogue of the beta integral holds. From Selberg's commentary [147], we know that in the 1940s he investigated finite field analogues of the more general Selberg integral, and formulated a conjecture that he could prove only for $n=2$. The existence of such finite field analogues was revealed by Selberg in the letter to Askey dated 25 March 1980, referred to on page 496. Selberg also mentioned this in some colloquium lectures. Ron Evans, a member of the audience on one of these occasions, has provided us with the following recollection [54]:

Somewhere around 1980, Selberg came to UCSD for a colloquium talk. Some department members at the UCSD talk were shocked by the subject matter. They were expecting to hear about his recent work, but instead his entire talk was on the Selberg integral. I was fascinated to learn of this integral, and ended up writing several papers on $q$-analogues and on finite field analogues. One of these (published in 1981) formulated $n$-dimensional finite field analogues, which I was able to prove for $n=2$. Selberg had mentioned in his talk that he had finite field analogues for $n=2$, so I was reluctant to write up my proof. However, some people who knew Selberg told me that he'd never ever get around to publishing his proof, so I took the bold step of asking permission to include my proof for $n=2$ with my general conjecture (with due credit, of course). He generously wrote back that he didn't mind if I publish a proof 
of the "right" version of the theorem, but that he didn't want to be credited with my version, which was too weak! So I proved the stronger theorem for $n=2$ that he supplied in his letter, and that led to stronger conjectures for general $n$ (ultimately proved by Anderson [2]).

The finite field paper of Evans referred to above is [49] (see also [55]), and the analogue of the Selberg integral proved by Anderson in [2] is

$$
\begin{aligned}
\mathcal{S}_{n}(\alpha, \beta, \gamma) & :=\sum_{f} \tau\left((-1)^{n \alpha} f^{\alpha}(0) f^{\beta}(1) \Delta_{f}^{\gamma}\right) \delta\left(\Delta_{f}\right) \\
& =\prod_{j=0}^{n-1} \frac{G^{*}(\alpha+j \gamma) G^{*}(\beta+j \gamma) G^{*}((j+1) \gamma)}{G^{*}(\alpha+\beta+(n+j-1) \gamma) G^{*}(\gamma)},
\end{aligned}
$$

for $\alpha, \beta, \gamma$ positive integers such that none of $\alpha+j \gamma, \beta+j \gamma$ and $\gamma+j \gamma$ is divisible by $q-1$ for $0 \leq j \leq n-1$. The sum on the left is over monic polynomials $f \in \mathbb{F}_{q}[x]$ of degree $n, \Delta_{f}$ is the discriminant of $f$, and $\delta(x)=\tau^{(q-1) / 2}(x)$. $G^{*}$ on the right is defined as $G^{*}(a)=q / G(-a)$, where $G$ is the Gauss sum (extended to all integers by $G(a)=G(a+q-1))$

$$
G(a)=\sum_{\zeta \in \mathbb{F}_{q}} \tau^{a}(\zeta) \psi(\zeta), \quad a=1,2, \ldots,
$$

with $\tau(0):=0$ and $\psi$ a nontrivial additive character. $\mathcal{S}_{1}$ is the Jacobi sum referred to at the beginning of this section, and (2.1) for $n=1$ is the analogue of the beta integral.

Anderson's paper left open some of the conjectures from [49], and Evans himself was able to apply Anderson's approach to provide the remaining proofs [50]. For a more comprehensive account of the finite field Selberg integral we refer the reader to [6].

In 1991, motivated by the quest for a proof of the finite field conjecture, G.W. Anderson 3] published a proof of the Selberg integral based on another multiple integral, namely

$$
\begin{aligned}
\int_{X} \prod_{1 \leq i<j \leq n}\left(t_{i}-t_{j}\right) \prod_{i=1}^{n} \prod_{j=1}^{n+1} \mid t_{i}- & \left.a_{j}\right|^{s_{j}-1} \mathrm{~d} t_{1} \cdots \mathrm{d} t_{n} \\
& =\frac{\prod_{i=1}^{n+1} \Gamma\left(s_{i}\right)}{\Gamma\left(\sum_{i=1}^{n+1} s_{i}\right)} \prod_{1 \leq i<j \leq n+1}\left(a_{i}-a_{j}\right)^{s_{i}+s_{j}-1},
\end{aligned}
$$

where $X$ is the domain of integration $a_{1}>t_{1}>a_{2}>t_{2}>\cdots>t_{n}>a_{n+1}$, and $\operatorname{Re}\left(s_{i}\right)>1$ for $1 \leq i \leq n+1$. Anderson's idea was to use (2.2) to compute in two different ways the integral

$$
\begin{aligned}
K(\alpha, \beta, \gamma):= & \int_{X^{\prime}} \prod_{i=1}^{n+1} x_{i}^{\alpha-1}\left(1-x_{i}\right)^{\beta-1} \prod_{i=1}^{n} \prod_{j=1}^{n+1}\left|y_{i}-x_{j}\right|^{\gamma-1} \\
& \times \prod_{1 \leq i<j \leq n}\left|y_{i}-y_{j}\right| \prod_{1 \leq i<j \leq n+1}\left|x_{i}-x_{j}\right| \mathrm{d} x_{1} \cdots \mathrm{d} x_{n+1} \mathrm{~d} y_{1} \cdots \mathrm{d} y_{n},
\end{aligned}
$$

where $X^{\prime}$ denotes the domain of integration

$$
1>x_{1}>y_{1}>x_{2}>y_{2}>\cdots>y_{n}>x_{n+1}>0 \text {. }
$$


First integrating over the $y$-variables gives

$$
K(\alpha, \beta, \gamma)=\frac{\Gamma^{n+1}(\gamma)}{(n+1) ! \Gamma((n+1) \gamma)} S_{n+1}(\alpha, \beta, \gamma)
$$

while first integrating over the $x$-variables gives

$$
K(\alpha, \beta, \gamma)=\frac{\Gamma(\alpha) \Gamma(\beta) \Gamma^{n}(\gamma)}{n ! \Gamma(\alpha+\beta+n \gamma)} S_{n}(\alpha+\gamma, \beta+\gamma, \gamma)
$$

Equating the two forms reveals a first order recurrence in $n$ for the Selberg integral. Together with the initial condition $S_{0}(\alpha, \beta, \gamma)=1$, this reclaims (1.1).

A large portion of Anderson's paper is devoted to a derivation of (2.2). This same multiple integral, written in the form

$$
\operatorname{det}_{1 \leq i, j \leq n}\left(\int_{a_{i+1}}^{a_{i}} t^{j-1} \prod_{l=1}^{n+1}\left|t-a_{l}\right|^{s_{l}-1} \mathrm{~d} t\right),
$$

was evaluated at around the same time by A. Varchenko [163, 164] in his work on hyperplane arrangements. That (2.3) is equal to the integral in (2.2) is a simple consequence of the Vandermonde determinant, a fact made explicit by D. Richards and Q. Zheng in [140].

Remarkably, in a 1998 paper by M.C. Bergère [21] proving a conjecture from the theory of Calogero-Sutherland models (see page 507) reference is made to (2.2), citing neither Anderson nor Varchenko, but a paper of A.L. Dixon [40] published in 1905! Indeed, consulting [40] reveals both (2.2) - obtained via essentially the same analysis as that used in [3] — and its equivalent determinant form (2.3).

2.2. Dotsenko-Fateev integrals. In the course of studies in conformal field theory, V.S. Dotsenko and V.A. Fateev [41] were led to consider the multiple integral

$$
\begin{array}{r}
\operatorname{PV} \int_{[0,1]^{p}[1, \infty)^{n-p}} \int_{[0,1]^{r}} \int_{[1, \infty)^{m-r}} \prod_{i=1}^{n} t_{i}^{\alpha}\left(1-t_{i}\right)^{\beta} \prod_{i=1}^{m} \tau_{i}^{\alpha^{\prime}}\left(\tau_{i}-1\right)^{\beta^{\prime}} \prod_{i=1}^{n} \prod_{j=1}^{m}\left(\tau_{j}-t_{i}\right)^{-2} \\
\times \prod_{1 \leq i<j \leq n}\left|t_{i}-t_{j}\right|^{2 \gamma} \prod_{1 \leq i<j \leq m}\left|\tau_{i}-\tau_{j}\right|^{2 \gamma^{\prime}} \mathrm{d} t_{1} \cdots \mathrm{d} t_{n} \mathrm{~d} \tau_{1} \cdots \mathrm{d} \tau_{m},
\end{array}
$$

where PV denotes the principal value, $\alpha / \alpha^{\prime}=\beta / \beta^{\prime}=-\gamma, \gamma \gamma^{\prime}=1$ and $0 \leq p \leq n$, $0 \leq r \leq m$. Note that the case $p=n$ and $m=0$ is, up to a shift by 1 in $\alpha$ and $\beta$, precisely the Selberg integral. Dotsenko and Fateev evaluated the above integral as a product of gamma and sine functions, reclaiming the Selberg integral as a special case.

The method employed by Dotsenko and Fateev for evaluating their integral suggests an approach [58] to the Selberg integral by studying the simpler $m=0$ case

$$
S_{n, p}(\alpha, \beta, \gamma):=\int_{[0,1]^{p}} \int_{[1, \infty)^{n-p}} \prod_{i=1}^{n} t_{i}^{\alpha-1}\left|1-t_{i}\right|^{\beta-1} \prod_{1 \leq i<j \leq n}\left|t_{i}-t_{j}\right|^{2 \gamma} \mathrm{d} t_{1} \cdots \mathrm{d} t_{n}
$$

for $0 \leq p \leq n$. Note that $S_{n, n}(\alpha, \beta, \gamma)=S_{n}(\alpha, \beta, \gamma)$, which is the Selberg integral. Also note that the change of variables $t_{i} \mapsto 1 / t_{i}$ for all $1 \leq i \leq n$ implies the transformation

$$
S_{n, p}(\alpha, \beta, \gamma)=S_{n, n-p}(1-\alpha-\beta-2(n-1) \gamma, \beta, \gamma)
$$


Singling out the integration variable $t_{p}$, viewing the integrand as an analytic function and replacing the interval $[0,1]$ by a contour along a positively oriented, indented semi-circle of infinite radius (with indentations at the branch points $t_{p}=$ $\left.0,1, t_{1}, \ldots, t_{p-1}, t_{p+1}, \ldots, t_{n}\right)$ yields the recurrence

$$
\begin{array}{r}
S_{n, p}(\alpha, \beta, \gamma)=\frac{p}{n-p+1} \frac{\sin \pi(n-p+1) \gamma \sin \pi(\alpha+\beta+(n+p-2) \gamma)}{\sin \pi p \gamma \sin \pi(\alpha+(p-1) \gamma)} \\
\times S_{n, p-1}(\alpha, \beta, \gamma) .
\end{array}
$$

Solving $S_{n, n}$ in terms of $S_{n, 0}$ and then using the transformation (2.5) to eliminate $S_{n, 0}$ in favour of $S_{n, n}$ gives the following functional equation for the Selberg integral

$$
S_{n}(\alpha, \beta, \gamma)=S_{n}(1-\alpha-\beta-2(n-1) \gamma, \beta, \gamma) \prod_{j=0}^{n-1} \frac{\sin \pi(\alpha+\beta+(n+j-1) \gamma)}{\sin \pi(\alpha+j \gamma)}
$$

The significance of this result is that it permits $S_{n}$, viewed as a function of $\alpha$, to be analytically continued to $\alpha \in \mathbb{C}$ with the exclusion of the zeros of $\sin \pi(\alpha+j \gamma)$. Indeed, for sufficiently small but positive values of $\operatorname{Re}(\beta)$ and $(n-1) \operatorname{Re}(\gamma)$, the Selberg integral requires $\operatorname{Re}(\alpha)>0$ but the transformed integral permits $\operatorname{Re}(\alpha)<$ $1-\operatorname{Re}(\beta)-2(n-1) \operatorname{Re}(\gamma)$, which is a bound greater than 0 . As a consequence, one can verify that $S_{n}(\alpha, \beta, \gamma)$ when divided by the right-hand side of (1.1) is a bounded analytic function in the complex $\alpha$-plane and is thus independent of $\alpha$. Symmetry then gives that this ratio is independent of $\beta$ as well. That the dependence on $\gamma$ and $n$ is correct is then verified by using

$$
\lim _{\alpha \rightarrow 0^{+}} \alpha S_{n}(\alpha, \beta, \gamma)=n S_{n-1}(2 \gamma-1, \beta, \gamma),
$$

a fact already noted and used for the same purpose in Selberg's original proof [145].

In their paper, Dotsenko and Fateev considered a further generalization of (1.1), referred to as the complex Selberg integral. This integral, which was also studied independently by K. Aomoto [8], can be written as an $n$-dimensional real integral with integration variables given by 2 -dimensional vectors

$$
A_{n}(\alpha, \beta, \gamma):=\int_{\mathbb{R}^{2}} \cdots \int_{\mathbb{R}^{2}} \prod_{i=1}^{n}\left|\vec{r}_{i}\right|^{2(\alpha-1)}\left|\vec{u}-\vec{r}_{i}\right|^{2(\beta-1)} \prod_{1 \leq i<j \leq n}\left|\vec{r}_{i}-\vec{r}_{j}\right|^{4 \gamma} \mathrm{d} \vec{r}_{1} \cdots \mathrm{d} \vec{r}_{n}
$$

where $\vec{u}$ is an arbitrary unity vector. Dotsenko and Fateev as well as Aomoto showed that up to a product of trigonometric functions the complex Selberg integral factors as a product of two Selberg integrals

$$
A_{n}(\alpha, \beta, \gamma)=S_{n}^{2}(\alpha, \beta, \gamma) \frac{1}{n !} \prod_{j=0}^{n-1} \frac{\sin \pi(\alpha+j \gamma) \sin \pi(\beta+j \gamma) \sin \pi(j+1) \gamma}{\sin \pi(\alpha+\beta+(n+j-1) \gamma) \sin \pi \gamma}
$$

provided (1.2) is supplemented by

$$
\operatorname{Re}(\alpha+\beta+(n-1) \gamma)<1 \text { and } \operatorname{Re}(\alpha+\beta+2(n-1) \gamma)<1 .
$$

K. Mimachi and M. Yoshida [120] (see also [119]) apply the theory of intersection numbers of twisted cycles to the conformal field theory study of Dotsenko and Fateev to give the evaluation of the product $S_{n}(\alpha, \beta, \gamma) S_{n}(-\alpha,-\beta,-\gamma)$, appropriately analytically continued. This is achieved without requiring the actual evaluation of the Selberg integral itself. 
2.3. $q$-Integrals and constant terms. Motivated by the Selberg integral and its success in dealing with Dyson- and Macdonald-type constant term identities, Askey in 1980, was led to consider several $q$-analogues of the Selberg integral and to study connections to $q$-constant term identities. In fact, one learns from [12] that he had earlier spent time searching for a proof of the Mehta integral upon its appearance in the problem section of the SIAM Review. To describe some of Askey's work, we require the multiple Jackson or $q$-integral

$$
\int_{0}^{1} \cdots \int_{0}^{1} f(t) \mathrm{d}_{q} t_{1} \cdots \mathrm{d}_{q} t_{n}:=(1-q)^{n} \sum_{k_{1}, \ldots, k_{n}=0}^{\infty} f\left(q^{k}\right) q^{k_{1}+\cdots+k_{n}}
$$

with $t=\left(t_{1}, \ldots, t_{n}\right), q^{k}=\left(q^{k_{1}}, \ldots, q^{k_{n}}\right)$ and $0<q<1$, and where it is assumed that the multiple sum on the right is absolutely convergent. Also needed is the $q$-shifted factorial

$$
(a ; q)_{z}=\prod_{j=0}^{\infty} \frac{1-a q^{j}}{1-a q^{z+j}}
$$

for $z \in \mathbb{C}$. Probably the most important of the $q$-Selberg integrals considered by Askey is

$$
\begin{aligned}
& S_{n}(\alpha, \beta, \gamma ; q) \\
& \quad:=\int_{0}^{1} \cdots \int_{0}^{1} \prod_{i=1}^{n} t_{i}^{\alpha-1}\left(q t_{i} ; q\right)_{\beta-1} \prod_{1 \leq i<j \leq n} t_{i}^{2 \gamma}\left(q^{1-\gamma} t_{j} / t_{i} ; q\right)_{2 \gamma} \mathrm{d}_{q} t_{1} \cdots \mathrm{d}_{q} t_{n} .
\end{aligned}
$$

It is immediate, at least formally, that

$$
\lim _{q \rightarrow 1^{-}} S_{n}(\alpha, \beta, \gamma ; q)=S_{n}(\alpha, \beta, \gamma) .
$$

For $\operatorname{Re}(\alpha)>0, \gamma$ a nonnegative integer, say $k$, and $\beta \in \mathbb{C}$ excluding the nonpositive integers, Askey conjectured (and proved for $k=2$ ) that [11, Conjecture 1]

$$
S_{n}(\alpha, \beta, k ; q)=q^{\alpha k\left(\begin{array}{l}
n \\
2
\end{array}\right)+2 k^{2}\left(\begin{array}{c}
n \\
3
\end{array}\right)} \prod_{j=0}^{n-1} \frac{\Gamma_{q}(\alpha+(j-1) k) \Gamma_{q}(\beta+(j-1) k) \Gamma_{q}(1+j k)}{\Gamma_{q}(\alpha+\beta+(n+j-2) k) \Gamma_{q}(1+k)},
$$

where $\Gamma_{q}(x)$ is the $q$-gamma function

$$
\Gamma_{q}(x)=\frac{(q ; q)_{x-1}}{(1-q)^{x-1}} .
$$

For Askey's other $q$-Selberg integrals and many further results relating to Jacksonintegral type extensions of beta integrals, see [5, 9, 10, 11, 51, 81, 84, 91, 166.

In 1988 Askey's conjecture was proved independently by L. Habsieger 74] and K. Kadell [85]. Both then applied the $q$-analogue of the identity (1.15) to (2.7) to obtain a $q$-generalization of the Morris integral (1.17). Expressing this integral as a constant term identity, they thus proved Morris' $q$-constant term conjecture [122]

$$
\begin{aligned}
\mathrm{CT} \prod_{j=1}^{n}\left(x_{j} ; q\right)_{a}\left(q / x_{j} ; q\right)_{b} \prod_{1 \leq i<j \leq n} & \left(x_{i} / x_{j} ; q\right)_{k}\left(q x_{j} / x_{i} ; q\right)_{k} \\
& =\prod_{j=0}^{n-1} \frac{\Gamma_{q}(1+a+b+j k) \Gamma_{q}(1+(j+1) k)}{\Gamma_{q}(1+a+j k) \Gamma_{q}(1+b+j k) \Gamma_{q}(1+k)} .
\end{aligned}
$$


When $a=b=0$, this is precisely the $\mathrm{A}_{n-1}$ case of the $q$-Macdonald constant term conjecture [108, Conjecture 3.1]

$$
\mathrm{CT} \prod_{\alpha \in \Phi^{+}} \prod_{i=1}^{k}\left(1-q^{i-1} \mathrm{e}^{-\alpha}\right)\left(1-q^{i} \mathrm{e}^{\alpha}\right)=\prod_{i=1}^{l}\left[\begin{array}{c}
d_{i} k \\
k
\end{array}\right]_{q}
$$

where

$$
\left[\begin{array}{l}
n \\
k
\end{array}\right]_{q}=\prod_{i=0}^{k} \frac{1-q^{n-k+i}}{1-q^{i}}
$$

is a $q$-binomial coefficient, and $\Phi$ is a reduced (finite) root system. To also include the root systems of type $\mathrm{BC}$, one again needs the numbers $s(\alpha)$ as defined above equation (1.22) [108, Conjecture 3.4]

$$
\mathrm{CT} \prod_{\alpha \in \Phi^{+}} \prod_{i=1}^{k}\left(1-q^{i s(\alpha)-1} \mathrm{e}^{-\alpha}\right)\left(1-q^{(i-1) s(\alpha)+1} \mathrm{e}^{\alpha}\right)=\prod_{i=1}^{l}\left[\begin{array}{c}
d_{i} k \\
k
\end{array}\right]_{q} .
$$

The $\mathrm{A}_{n-1}$ case of (2.8), was in fact proved prior to the work of Habsieger and Kadell by Zeilberger and D.M. Bressoud [174], who proved the more general $q$ Dyson conjecture

$$
\mathrm{CT} \prod_{1 \leq i<j \leq n}\left(\frac{x_{i}}{x_{j}}\right)_{a_{i}}\left(\frac{q x_{j}}{x_{i}}\right)_{a_{j}}=\frac{(q ; q)_{a_{1}+\cdots+a_{n}}}{(q ; q)_{a_{1}} \cdots(q ; q)_{a_{n}}}
$$

formulated by G.E. Andrews [4].

R.A. Gustafson [71], at around the same time as Anderson's work on the DixonAnderson integral, made use of a further $q$-generalization of (2.2) and invented the same general strategy as used in [3] to derive the $\mathrm{BC}_{n}$-type constant term identity

$$
\mathrm{CT} \Delta\left(x ; t, t_{1}, \ldots, t_{4}\right)=2^{n} n ! \prod_{j=1}^{n} \frac{(t ; q)_{\infty}\left(t^{n+j-2} t_{1} t_{2} t_{3} t_{4} ; q\right)_{\infty}}{\left(t^{j} ; q\right)_{\infty}(q ; q)_{\infty} \prod_{1 \leq r<s \leq 4}\left(t^{j-1} t_{r} t_{s} ; q\right)_{\infty}}
$$

where

$$
\begin{aligned}
\Delta\left(x ; t, t_{1}, \ldots, t_{4}\right) & :=\prod_{i=1}^{n} \frac{\left(x_{i}^{2} ; q\right)_{\infty}\left(x_{i}^{-2} ; q\right)_{\infty}}{\prod_{r=1}^{4}\left(t_{r} x_{i} ; q\right)_{\infty}\left(t_{r} x_{i}^{-1} ; q\right)_{\infty}} \\
& \times \prod_{1 \leq i<j \leq n} \frac{\left(x_{i} x_{j}^{-1} ; q\right)_{\infty}\left(x_{i}^{-1} x_{j} ; q\right)_{\infty}\left(x_{i} x_{j} ; q\right)_{\infty}\left(x_{i}^{-1} x_{j}^{-1} ; q\right)_{\infty}}{\left(t x_{i} x_{j}^{-1} ; q\right)_{\infty}\left(t x_{i}^{-1} x_{j} ; q\right)_{\infty}\left(t x_{i} x_{j} ; q\right)_{\infty}\left(t x_{i}^{-1} x_{j}^{-1} ; q\right)_{\infty}} .
\end{aligned}
$$

This is a generalization of the so-called Macdonald-Morris constant term identity, and it implies the $\mathrm{B}_{n}, \mathrm{C}_{n}, \mathrm{D}_{n}$ and $\mathrm{BC}_{n}$ cases of (2.8) through specialisation [71].

Most other cases of (2.8) were proved on a case by case basis, often using methods based on $q$-integrals of Selberg type [51, 65, 66, 73, 87, 156, 175, 176, 177], but the three exceptional root systems $\mathrm{E}_{6}, \mathrm{E}_{7}$ and $\mathrm{E}_{8}$ refused to surrender until I. Cherednik gave a uniform proof for all reduced root systems based on his theory of double affine Hecke algebras [29, 30, 31, 111].

\section{The Selberg integral and multivariable orthogonal polynomials}

3.1. Jack polynomial theory. It has been known since the early 1970s 26, 159, 160] that (1.9) with $\beta=2 \gamma$ - to be denoted $\exp (-2 \gamma W)$ in analogy with (1.7) - is 
the absolute value squared of the ground-state wave function for the Schrödinger operator

$$
H=-\sum_{i=1}^{n} \frac{\partial^{2}}{\partial \theta_{i}^{2}}+\frac{1}{2} \gamma(\gamma-1) \sum_{1 \leq i<j \leq n} \frac{1}{\sin ^{2}\left(\frac{1}{2}\left(\theta_{i}-\theta_{j}\right)\right)} .
$$

This operator, known as the Calogero-Sutherland Hamiltonian, describes a system of $n$ identical quantum particles on the unit circle, with $\theta_{i} \in[0,2 \pi)$ for $1 \leq i \leq n$ the (angular) positions of the particles. The interaction between the particles is described by a $1 / r^{2}$ two-body potential, $2\left|\sin \left(\left(\theta_{i}-\theta_{j}\right) / 2\right)\right|$ being the cord-length between particles located at $\theta_{i}$ and $\theta_{j}$.

B. Sutherland [159] showed that the eigenvalue $E_{0}$ corresponding to the groundstate wave function is given by $E_{0}=n\left(n^{2}-1\right) \gamma^{2} / 12$. Subsequently, he showed [160] that the conjugated operator

$$
\mathrm{e}^{\gamma W}\left(H-E_{0}\right) \mathrm{e}^{-\gamma W}=\sum_{i=1}^{n}\left(x_{i} \frac{\partial}{\partial x_{i}}\right)^{2}+2 \gamma \sum_{\substack{i, j=1 \\ i \neq j}}^{n} \frac{x_{i}+x_{j}}{x_{i}-x_{j}} \frac{\partial}{\partial x_{i}},
$$

where $x_{j}:=\exp \left(\mathrm{i} \theta_{j}\right)$, admits a complete set of symmetric polynomial eigenfunctions $P_{\lambda}^{(1 / \gamma)}(x)$. These polynomials, now referred to as Jack polynomials, depend on $x=\left(x_{1}, \ldots, x_{n}\right)$ and are indexed by partitions $\lambda$ of at most $n$ parts; $\lambda=\left(\lambda_{1}, \ldots, \lambda_{n}\right)$ with $\lambda_{1} \geq \lambda_{2} \geq \cdots \geq \lambda_{n} \geq 0$. With $m_{\lambda}$ denoting the monomial symmetric function indexed by $\lambda$ and $<$ the dominance ordering on partitions, the Jack polynomials have the structure

$$
P_{\lambda}^{(1 / \gamma)}(x)=m_{\lambda}(x)+\sum_{\mu<\lambda} a_{\lambda \mu} m_{\mu}(x)
$$

for some coefficients $a_{\lambda \mu}=a_{\lambda \mu}(\gamma)$.

One fundamental property of the Jack polynomials is that they are orthogonal with respect to the inner product

$$
\langle f, g\rangle_{\gamma}:=\frac{1}{(2 \pi)^{n}} \int_{-\pi}^{\pi} \cdots \int_{-\pi}^{\pi} f\left(\mathrm{e}^{\mathrm{i} \theta}\right) g\left(\mathrm{e}^{-\mathrm{i} \theta}\right) \prod_{1 \leq i<j \leq n}\left|\mathrm{e}^{\mathrm{i} \theta_{i}}-\mathrm{e}^{\mathrm{i} \theta_{j}}\right|^{2 \gamma} \mathrm{d} \theta_{1} \cdots \mathrm{d} \theta_{n}
$$

where $f\left(\mathrm{e}^{\mathrm{i} \theta}\right)=f\left(\mathrm{e}^{\mathrm{i} \theta_{1}}, \ldots, \mathrm{e}^{\mathrm{i} \theta_{n}}\right)$. To state the orthogonality as well as the quadratic norm evaluation, let

$$
[b]_{\lambda}^{(\gamma)}=\prod_{i \geq 1}(b+(1-i) \gamma)_{\lambda_{i}}
$$

with $(b)_{n}=b(b+1) \cdots(b+n-1)$ a Pochhammer symbol. Also let $c_{\lambda}(\gamma)$ and $c_{\lambda}^{\prime}(\gamma)$ be given by

$$
\begin{aligned}
& c_{\lambda}(\gamma)=\prod_{s \in \lambda}(a(s)+l(s) \gamma+\gamma), \\
& c_{\lambda}^{\prime}(\gamma)=\prod_{s \in \lambda}(a(s)+l(s) \gamma+1),
\end{aligned}
$$

where $a(s)$ and $l(s)$ are the arm-length and leg-length of the square $s$ in the diagram of the partition $\lambda$, and $|\lambda|$ is the total number of boxes in the diagram of $\lambda[110]$. Then

$$
\left\langle P_{\lambda}^{(1 / \gamma)}, P_{\mu}^{(1 / \gamma)}\right\rangle_{\gamma}=\delta_{\lambda \mu} \frac{c_{\lambda}^{\prime}(\gamma)}{[1+(n-1) \gamma]_{\lambda}^{(\gamma)}} \frac{\Gamma(1+n \gamma)}{\Gamma^{n}(1+\gamma)} P_{\lambda}^{(1 / \gamma)}\left(1^{n}\right)
$$


where $\delta_{\lambda \mu}$ is the Kronecker delta function and $\left(1^{n}\right)$ is shorthand for $(1,1, \ldots, 1)$. The orthogonality relation is consistent with, but not an immediate consequence of, the operator (3.1) being self-adjoint with respect to (3.3). The complication is that not all eigenvalues of (3.1) are distinct. This degeneracy can be removed by introducing the mutually commuting Cherednik operators $\xi_{i}$ for $1 \leq i \leq n$ [28, 43],

$$
\xi_{i}=1-i+\frac{x_{i}}{\gamma} \frac{\partial}{\partial x_{i}}+\sum_{j=1}^{i-1} \frac{x_{i}}{x_{i}-x_{j}}\left(1-s_{i j}\right)+\sum_{j=i+1}^{n} \frac{x_{j}}{x_{i}-x_{j}}\left(1-s_{i j}\right),
$$

where $s_{i j}$ acts by permutation $x_{i}$ and $x_{j}$ and 1 represents the identity operator. Any symmetric combination of the $\xi_{i}$, and in particular $\prod_{i=1}^{n}\left(1-u \xi_{i}\right)$, has the Jack polynomials as simultaneous eigenfunctions.

The Cherednik operators can be used to construct the Jack polynomial shift operator - a special case of the shift operators studied by Heckman and Opdam, and used by the latter to prove the Macdonald integral and constant term conjectures. Properties of the Jack shift operator not only imply (1.12) or, equivalently, (1.13), but also the more general quadratic norm evaluation of the Jack polynomials corresponding to (3.5) with $\lambda=\mu$ [89]. (For $\lambda=0$ this yields (1.12).) With $\Delta(x)$ the Vandermonde product (1.21) and $Y_{ \pm}:=\gamma^{n(n-1) / 2} \prod_{1 \leq i<j \leq n}\left(\xi_{i}-\xi_{j} \mp 1\right)$, the Jack shift operators are defined by $G_{+}:=\Delta^{-1} Y_{+}, G_{-}=Y_{-} \Delta$. They have an adjoint-type property with respect to the inner product (3.3),

$$
\left\langle G_{+} f, g\right\rangle_{\gamma+1}=\left\langle f, G_{-} g\right\rangle_{\gamma} .
$$

Also, with

$$
a_{\lambda}^{ \pm}(\gamma)=\prod_{1 \leq i<j \leq n}\left(\lambda_{i}-\lambda_{j} \pm 1+(j-i \mp 1) \gamma\right)
$$

and $\delta$ the staircase partition $(n-1, n-2, \ldots, 1,0)$, the shift operators act on the Jack polynomials as

$$
\begin{aligned}
G_{+} P_{\lambda+\delta}^{(1 / \gamma)} & =a_{\lambda}^{+}(\gamma+1) P_{\lambda}^{(1 /(\gamma+1))}, \\
G_{-} P_{\lambda}^{(1 /(\gamma+1))} & =a_{\lambda}^{-}(\gamma+1) P_{\lambda+\delta}^{(1 / \gamma)} .
\end{aligned}
$$

It follows from (3.6) and (3.8) that

$$
\left\langle P_{\lambda}^{(1 /(\gamma+1))}, P_{\lambda}^{(1 /(\gamma+1))}\right\rangle_{\gamma+1}=\frac{a_{\lambda}^{-}(\gamma+1)}{a_{\lambda}^{+}(\gamma+1)}\left\langle P_{\lambda+\delta}^{(1 / \gamma)}, P_{\lambda+\delta}^{(1 / \gamma)}\right\rangle_{\gamma}
$$

and thus

$$
\left\langle P_{\lambda}^{(1 /(\gamma+k))}, P_{\lambda}^{(1 /(\gamma+k))}\right\rangle_{\gamma+k}=\left\langle P_{\lambda+k \delta}^{(1 / \gamma)}, P_{\lambda+k \delta}^{(1 / \gamma)}\right\rangle_{\gamma} \prod_{j=0}^{k-1} \frac{a_{\lambda+j \delta}^{-}(\gamma+k-j)}{a_{\lambda+j \delta}^{+}(\gamma+k-j)} .
$$

Taking $\gamma=0$, using that $P_{\lambda}^{(\infty)}=m_{\lambda}$ (the monomial symmetric function) and

$$
\left\langle m_{\mu}, m_{\mu}\right\rangle_{0}=\mathrm{CT}\left(m_{\mu}(x) m_{\mu}\left(x^{-1}\right)\right)=m_{\mu}\left(1^{n}\right),
$$

which is $n$ ! for $\mu=\lambda+k \delta$, it follows that for nonnegative integer $k$

$$
\left\langle P_{\lambda}^{(1 / k)}, P_{\lambda}^{(1 / k)}\right\rangle_{k}=n ! \prod_{j=0}^{k-1} \frac{a_{\lambda+j k}^{-}(k-j)}{a_{\lambda+j k}^{+}(k-j)} .
$$


Using the evaluation formula 152]

$$
P_{\lambda}^{(1 / \gamma)}\left(1^{n}\right)=\frac{[n \gamma]_{\lambda}^{(\gamma)}}{c_{\lambda}(\gamma)}
$$

and the definitions (3.4) and (3.7), it is now a straightforward exercise to verify that for $\gamma=k$ (3.5) coincides with (3.9). Analytic continuation off the integers is then required to establish (3.5) for all $\operatorname{Re}(\gamma)>-1 / n$.

A further fundamental property of the Jack polynomials is R.P. Stanley's Cauchy identity [152],

$$
\sum_{\lambda} \frac{c_{\lambda}(\gamma)}{c_{\lambda}^{\prime}(\gamma)} P_{\lambda}^{(1 / \gamma)}(x) P_{\lambda}^{(1 / \gamma)}(y)=\prod_{i=1}^{n} \prod_{j=1}^{m}\left(1-x_{i} y_{j}\right)^{-\gamma},
$$

where $x=\left(x_{1}, \ldots, x_{n}\right), y=\left(y_{1}, \ldots, y_{m}\right)$. Setting $y=\left(1^{m}\right)$, using the evaluation formula (3.10) with $n \mapsto m$ and a standard analytic argument to replace $m$ by $a \alpha$, leads to Z. Yan's binomial theorem for Jack polynomials [173],

$$
\sum_{\lambda} \frac{[a]_{\lambda}^{(\alpha)}}{c_{\lambda}^{\prime}(\alpha)} P_{\lambda}^{(\alpha)}(x)=\prod_{i=1}^{n} \frac{1}{\left(1-x_{i}\right)^{a}}
$$

This, together with the orthogonality (3.5), the property

$$
P_{\left(\lambda_{1}+a, \ldots, \lambda_{n}+a\right)}^{(\alpha)}(x)=\left(x_{1} \cdots x_{n}\right)^{a} P_{\lambda}^{(\alpha)}(x), \quad a=0,1,2, \ldots
$$

and the gamma reflection formula, implies a generalization of the Morris integral (1.17) [17]

$$
\begin{aligned}
& \frac{1}{(2 \pi)^{n}} \int_{-\pi}^{\pi} \cdots \int_{-\pi}^{\pi} P_{\lambda}^{(1 / \gamma)}\left(-\mathrm{e}^{\mathrm{i} \theta}\right) \prod_{i=1}^{n} \mathrm{e}^{\frac{1}{2} \mathrm{i} \theta_{i}(a-b)}\left|1+\mathrm{e}^{\mathrm{i} \theta_{i}}\right|^{a+b} \\
& \times \prod_{1 \leq i<j \leq n}\left|\mathrm{e}^{\mathrm{i} \theta_{i}}-\mathrm{e}^{\mathrm{i} \theta_{j}}\right|^{2 \gamma} \mathrm{d} \theta_{1} \cdots \mathrm{d} \theta_{n} \\
&=\frac{[-b]_{\lambda}^{(\gamma)}}{[1+a+(n-1) \gamma]_{\lambda}^{(\gamma)}} P_{\lambda}^{(1 / \gamma)}\left(1^{n}\right) M_{n}(a, b, \gamma) .
\end{aligned}
$$

Applying (1.15) finally results in a generalization of the Selberg integral

$$
\begin{array}{r}
\int_{0}^{1} \cdots \int_{0}^{1} P_{\lambda}^{(1 / \gamma)}(t) \prod_{i=1}^{n} t_{i}^{\alpha-1}\left(1-t_{i}\right)^{\beta-1} \prod_{1 \leq i<j \leq n}\left|t_{i}-t_{j}\right|^{2 \gamma} \mathrm{d} t_{1} \cdots \mathrm{d} t_{n} \\
=\frac{[\alpha+(n-1) \gamma]_{\lambda}^{(\gamma)}}{[\alpha+\beta+2(n-1) \gamma]_{\lambda}^{(\gamma)}} P_{\lambda}^{(1 / \gamma)}\left(1^{n}\right) S_{n}(\alpha, \beta, \gamma) .
\end{array}
$$

This evaluation is usually referred to as Kadell's integral [88] after its first prover, but as a conjecture it is due to Macdonald [109, Conjecture (C5)]. When $\lambda=\left(1^{r}\right)$, in which case the Jack polynomial is nothing but the $r$ th elementary symmetric function, the above is known as Aomoto's integral, who used it in [7] to give what is arguably the first elementary proof of the Selberg integral. A proof of Kadell's integral along the lines of Anderson's proof of the Selberg integral as described on 
pages 502-503 has recently been obtained in [167 through use of the OkounkovOlshanski integral formula for Jack polynomials [126],

$$
\begin{aligned}
P_{\lambda}^{(1 / \gamma)}(x)= & \prod_{i=1}^{n-1} \frac{\Gamma\left(\lambda_{i}+(n-i+1) \gamma\right)}{\Gamma\left(\lambda_{i}+(n-i) \gamma\right) \Gamma(\gamma)} \prod_{1 \leq i<j \leq n}\left(x_{j}-x_{i}\right)^{1-2 \gamma} \\
& \times \int_{Y} P_{\lambda}^{(1 / \gamma)}(y) \prod_{1 \leq i<j \leq n-1}\left(y_{j}-y_{i}\right) \prod_{i=1}^{n-1} \prod_{j=1}^{n}\left|y_{i}-x_{j}\right|^{\gamma-1} \mathrm{~d} y_{1} \cdots \mathrm{d} y_{n-1},
\end{aligned}
$$

where $Y$ denotes the domain $x_{1}<y_{1}<x_{2}<\cdots<x_{n-1}<y_{n-1}<x_{n}$ and $\lambda$ is a partition of at most $n-1$ parts.

An open problem, settled only in the 2-variable case [53], is to find (and prove) a finite field analogue of Kadell's integral.

The binomial theorem for Jack polynomials (3.12) can be written succinctly in hypergeometric notation as

$$
{ }_{1} F_{0}(\gamma)\left({ }_{-}^{a} ; x\right)=\prod_{i=1}^{n} \frac{1}{\left(1-x_{i}\right)^{a}},
$$

where ${ }_{1} F_{0}^{(\gamma)}$ is an example of a hypergeometric function with the Jack polynomial argument

$$
{ }_{r} F_{s}^{(\gamma)}\left(\begin{array}{c}
a_{1}, \ldots, a_{r} \\
b_{1}, \ldots, b_{s}
\end{array} ; x\right):=\sum_{\lambda} \frac{\left[a_{1}\right]_{\lambda}^{(\gamma)} \cdots\left[a_{r}\right]_{\lambda}^{(\gamma)}}{\left[b_{1}\right]_{\lambda}^{(\gamma)} \cdots\left[b_{s}\right]_{\lambda}^{(\gamma)}} \frac{P_{\lambda}^{(1 / \gamma)}(x)}{c_{\lambda}^{\prime}(\gamma)}
$$

When $n=1$, so that $x$ is a scalar, this function reduces to the classical hypergeometric function ${ }_{r} F_{s}$. For general $n$, hypergeometric functions of the type (3.14) have their genesis in the work of A.G. Constantine 33, C.S. Herz 77] and R.J. Muirhead [123], but were first studied in their full form presented here by Kaneko 90], A. Korányi [101] and Yan [173]. The case $r=2, s=1$ of (3.14) shares many properties in common with its $n=1$ counterpart, the Gauss hypergeometric function.

One such property is that ${ }_{2} F_{1}(\gamma)$ solves the $n$-dimensional analogue of Euler's hypergeometric equation. Specifically, Yan [173] and Kaneko [90] independently showed that ${ }_{2} F_{1}(\gamma)(a, b ; c ; x)$ is the unique symmetric function, analytic at the origin, that solves the system of $n$ partial differential equations

$$
\begin{aligned}
x_{i}\left(1-x_{i}\right) \frac{\partial^{2} F}{\partial x_{i}^{2}}+(c- & \left.(n-1) \gamma-(a+b+1-(n-1) \gamma) x_{i}\right) \frac{\partial F}{\partial x_{i}}-a b F \\
& +\gamma \sum_{\substack{j=1 \\
j \neq i}}^{n} \frac{1}{x_{i}-x_{j}}\left(x_{i}\left(1-x_{i}\right) \frac{\partial F}{\partial x_{i}}-x_{j}\left(1-x_{j}\right) \frac{\partial F}{\partial x_{j}}\right)=0
\end{aligned}
$$

for $1 \leq i \leq n$.

In the one-variable theory, the Gauss hypergeometric function admits an integral representation due to Euler [48],

$$
{ }_{2} F_{1}\left(\begin{array}{c}
a, b \\
c
\end{array} ; z\right)=\frac{\Gamma(c)}{\Gamma(b) \Gamma(c-b)} \int_{0}^{1} t^{b-1}(1-t)^{c-b-1}(1-z t)^{-a} \mathrm{~d} t
$$

for $\operatorname{Re}(c)>\operatorname{Re}(b)>0$ with a branch cut in the complex $z$-plane from 1 to infinity. When $z=1$, the integral on the right is the beta integral (1.3), resulting in a closed form evaluation of the ${ }_{2} F_{1}$ due to Gauss. In the multivariable theory an analogous 
results holds, where now the key integral-evaluation is provided by the Selberg integral. Multiplying both sides of Kadell's integral by $z^{|\lambda|}[a]_{\lambda}^{(\gamma)} / c_{\lambda}^{\prime}(\gamma)$, summing the left-hand side using the binomial theorem (3.12), and using the definition (3.14) on the right-hand side, shows that Euler's integral extends to [90]

$$
\begin{aligned}
& { }_{2} F_{1}(\gamma)\left(\begin{array}{c}
a, b \\
c
\end{array} ;\left(z^{n}\right)\right)=\prod_{j=0}^{n-1} \frac{\Gamma(c-j \gamma) \Gamma(1+\gamma)}{\Gamma(b-j \gamma) \Gamma(c-b-j \gamma) \Gamma(1+(j+1) \gamma)} \\
& \times \int_{0}^{1} \cdots \int_{0}^{1} \prod_{i=1}^{n} t_{i}^{\alpha-1}\left(1-t_{i}\right)^{\beta-1}\left(1-z t_{i}\right)^{-a} \prod_{1 \leq i<j \leq n}\left|t_{i}-t_{j}\right|^{2 \gamma} \mathrm{d} t_{1} \cdots \mathrm{d} t_{n},
\end{aligned}
$$

with $\alpha=b-(n-1) \gamma$ and $\beta=c-b-(n-1) \gamma$. Evaluating the $z=1$ instance of the integral by the Selberg integral (which, incidentally, follows by taking $z=0$ in (3.16)) implies a generalized Gauss summation [173]

$$
{ }_{2} F_{1}^{(\gamma)}\left(\begin{array}{c}
a, b \\
c
\end{array} ;\left(1^{n}\right)\right)=\prod_{j=0}^{n-1} \frac{\Gamma(c-j \gamma) \Gamma(c-a-b-j \gamma)}{\Gamma(c-a-j \gamma) \Gamma(c-b-j \gamma)} .
$$

The normalized integrand of the Selberg integral is referred to as the Selberg density. The Cauchy identity (3.11) gives rise to a special limiting case of this density known as the Laguerre PDF 61, 82]. Before describing this result, we first remark that the Jack polynomial at $\gamma=1$ is equal to the Schur polynomial $s_{\lambda}$ while $c_{\lambda}(1) / c_{\lambda}^{\prime}(1)=1$. The normalized summand of (3.11) then reads

$$
s_{\lambda}(x) s_{\lambda}(y) \prod_{i=1}^{n} \prod_{j=1}^{m}\left(1-x_{i} y_{j}\right),
$$

which may be recognized as the measure on partitions induced by the RobinsonSchensted-Knuth correspondence, see, e.g., [83, 128]. As such, the Schur measure holds a special place in certain studies relating to the representation theory of the symmetric group [25, 138].

For general $\gamma$ the normalized summand of (3.11) implies the more general measure on partitions

$$
\frac{c_{\lambda}(\gamma)}{c_{\lambda}^{\prime}(\gamma)} P_{\lambda}^{(1 / \gamma)}(x) P_{\lambda}^{(1 / \gamma)}(y) \prod_{i=1}^{n} \prod_{j=1}^{m}\left(1-x_{i} y_{j}\right)^{\gamma},
$$

where $n \leq m$ and $\lambda$ is a partition of at most $n$ parts. To obtain the Laguerre PDF [61, 82] one needs to specialize $x$ and $y$ in (3.17) to

$$
x_{i}=q^{1 / 2}, \quad 1 \leq i \leq n \quad \text { and } \quad y_{j}=q^{1 / 2}, \quad 1 \leq j \leq m .
$$

Use of the Jack polynomial evaluation formula (3.10) allows all terms in (3.17) to be made explicit. The remaining step is to take the scaling limit, turning the discrete measure on partitions into a continuous one on the positive real line. This is done by setting $q=\exp (-1 / L)$, introducing the scaled variables $t_{j}:=\lambda_{j} / L$ and then taking the large $L$ limit for fixed $t_{j}$. One finds that (3.17) multiplied by $L^{n}$ tends to the PDF, supported on $t_{1}>t_{2}>\cdots>t_{n}>0$,

$$
\frac{1}{W_{n}((m-n+1) \gamma, \gamma)} \prod_{i=1}^{n} \mathrm{e}^{-t_{i}} t_{i}^{(m-n+1) \gamma-1} \prod_{1 \leq i<j \leq n}\left(t_{i}-t_{j}\right)^{2 \gamma},
$$


where $W_{n}$ is the normalization

$$
W_{n}(\alpha, \gamma)=\frac{1}{n !} \prod_{j=0}^{n-1} \frac{\Gamma(\alpha+j \gamma) \Gamma((j+1) \gamma)}{\Gamma(\gamma)}
$$

To obtain this same result as a limit of the Selberg density, order the integration variables in the latter and write $\beta=L$. Then the change of variables $t_{i} \mapsto t_{i} / L$ followed by the limit $L \rightarrow \infty$ results in (3.18) after identifying $\alpha$ with $(m-n+1) \gamma$. This limiting "Laguerre" case of the Selberg integral, leading to the evaluation (3.19), is contained as equation (1) in the letter from Selberg to Dyson reprinted on page 497.

Askey and Richards [13] (see also [115]) have shown that after some fairly straightforward manipulations and a change of variables, the Laguerre limit of the Selberg integral leads to the evaluation

$$
\begin{aligned}
\int_{D} \prod_{i=1}^{n} t_{i}^{\alpha-1}\left(1-\sum_{i=1}^{n} t_{i}\right)^{\beta-1} \prod_{1 \leq i<j \leq n}\left|t_{i}-t_{j}\right|^{2 \gamma} \mathrm{d} t_{1} \cdots \mathrm{d} t_{n} \\
=\frac{\Gamma(\beta)}{\Gamma(\alpha n+\beta+n(n-1) \gamma)} \prod_{j=0}^{n-1} \frac{\Gamma(\alpha+j \gamma) \Gamma(1+(j+1) \gamma)}{\Gamma(1+\gamma)}
\end{aligned}
$$

where $D$ is the domain $t_{i} \geq 0$ for $1 \leq i \leq n$ such that $t_{1}+\cdots+t_{n} \leq 1$, and

$$
\operatorname{Re}(\alpha)>0, \operatorname{Re}(\beta)>0, \operatorname{Re}(\gamma)>-\min \{1 / n, \operatorname{Re}(\alpha) /(n-1)\} .
$$

According to Askey and Richards, the first statement of (3.20) is due to Selberg at a meeting held in Sri Lanka during December 1987. The intriguing point is that while Selberg did not give his derivation of (3.20), he is reported to have said that it was different from (1.1), and had the advantage of working in the finite field case. The derivation given in [13] does not work in the case of a finite field, and so must be different from the one that was known to Selberg.

Finally, returning to Askey's $q$-Selberg integral of Section 2.3, we remark that the density function corresponding to the normalized integrand of (2.7) can be deduced from Macdonald polynomial theory [110] following a procedure similar to that of deducing (3.18) from (3.17) 61]. Macdonald polynomials $P_{\lambda}(x ; q, t)$ are generalizations of Jack polynomials - the latter being reclaimed according to $\lim _{q \rightarrow 1} P_{\lambda}\left(x ; q, q^{\gamma}\right)=P_{\lambda}^{(1 / \gamma)}(x)$ - exhibiting the structure (3.2) and the orthogonality

$$
\int_{-\pi}^{\pi} \cdots \int_{-\pi}^{\pi} P_{\lambda}\left(\mathrm{e}^{i \theta} ; q, t\right) P_{\mu}\left(\mathrm{e}^{-i \theta} ; q, t\right) \prod_{1 \leq i<j \leq n} \frac{\left(\mathrm{e}^{i\left(\theta_{i}-\theta_{j}\right)} ; q\right)_{\infty}}{\left(t \mathrm{e}^{i\left(\theta_{i}-\theta_{j}\right)} ; q\right)_{\infty}} \mathrm{d} \theta_{1} \cdots \mathrm{d} \theta_{n} \propto \delta_{\lambda \mu} .
$$

It is the connection between affine Hecke algebras and Macdonald type orthogonal polynomials that is at the heart of Cherednik's proof of the $q$-constant term conjectures for arbitrary root systems [29, 30, 31, 111] as mentioned at the end of Section 2.3 .

3.2. Classical multivariable orthogonal polynomials. By the change of variables $t=(1-x) / 2$ and a shift in $\alpha$ and $\beta$ by 1 , the Euler beta integral (1.3) takes the form

$$
J(\alpha, \beta):=\int_{-1}^{1}(1-x)^{\alpha}(1+x)^{\beta} \mathrm{d} x=2^{\alpha+\beta+1} \frac{\Gamma(\alpha+1) \Gamma(\beta+1)}{\Gamma(\alpha+\beta+1)} .
$$


The integrand on the left is the weight function of the Jacobi polynomials $P_{n}^{(\alpha, \beta)}(x)$ [6, 80]. Up to normalization these are the unique functions, analytic around the origin, solving the second-order ODE

$$
\left(1-x^{2}\right) y^{\prime \prime}+(\beta-\alpha-x(\alpha+\beta+2)) y^{\prime}+n(n+\alpha+\beta+1) y=0 .
$$

Defining the inner product

$$
\langle f, g\rangle_{\alpha, \beta}:=\int_{-1}^{1} f(x) g(x)(1-x)^{\alpha}(1+x)^{\beta} \mathrm{d} x,
$$

the Jacobi polynomials satisfy the orthogonality relation

$$
\left\langle P_{n}^{(\alpha, \beta)}, P_{m}^{(\alpha, \beta)}\right\rangle_{\alpha, \beta}=\delta_{m, n} \frac{(\alpha+1)_{n}(\beta+1)_{n}}{n !(\alpha+\beta+1)_{n}} \frac{\alpha+\beta+1}{\alpha+\beta+2 n+1} J(\alpha, \beta) .
$$

All other classical orthogonal polynomials, such as the Laguerre and Hermite polynomials (corresponding to weights $x^{\alpha} \exp (-x)$ and $\exp \left(-x^{2}\right)$, respectively) follow from the Jacobi polynomials by taking appropriate limits.

Several people have studied multivariable generalizations of the Jacobi, Laguerre and Hermite polynomials [16, 36, 38, 44, 102, 103, 104, 112, 131]. The most general of these are the multivariable Jacobi polynomials $P_{\lambda}^{(\alpha, \beta, \gamma)}(x)$ which arise as the eigenfunctions of the operator

$$
\sum_{i=1}^{n}\left(\left(1-x_{i}^{2}\right) \frac{\partial^{2}}{\partial x_{i}^{2}}+\left(\beta-\alpha-x_{i}(\alpha+\beta+2)\right) \frac{\partial}{\partial x_{i}}\right)+2 \gamma \sum_{\substack{i, j=1 \\ i \neq j}}^{n} \frac{1-x_{i}^{2}}{x_{i}-x_{j}} \frac{\partial}{\partial x_{i}}
$$

The $P_{\lambda}^{(\alpha, \beta, \gamma)}(x)$ are orthogonal with respect to an inner product with weight function derived from the Selberg integral. With

$$
\langle f, g\rangle_{\alpha, \beta, \gamma}:=\int_{[-1,1]^{n}} f(x) g(x) \prod_{i=1}^{n}\left(1-x_{i}\right)^{\alpha}\left(1+x_{i}\right)^{\beta} \prod_{1 \leq i<j \leq n}\left|x_{i}-x_{j}\right|^{2 \gamma} \mathrm{d} x_{1} \cdots \mathrm{d} x_{n}
$$

for $x=\left(x_{1}, \ldots, x_{n}\right)$, the multivariable Jacobi polynomials satisfy

$$
\left\langle P_{\lambda}^{(\alpha, \beta, \gamma)}, P_{\mu}^{(\alpha, \beta, \gamma)}\right\rangle_{\alpha, \beta, \gamma}=0 \quad \text { if } \lambda \neq \mu .
$$

The quadratic norm evaluation can be computed explicitly in term of Pochhammer symbols and gamma functions using the shift operators of Heckman and Opdam [129]. From the Selberg integral it of course immediately follows that

$$
\langle 1,1\rangle_{\alpha, \beta, \gamma}=2^{n(\alpha+\beta+1+(n-1) \gamma)} S_{n}(\alpha+1, \beta+1, \gamma) .
$$

Two important limiting cases of the inner product 3.21) are

$$
\langle f, g\rangle_{\gamma}:=\int_{\mathbb{R}^{n}} f(x) g(x) \prod_{i=1}^{n} \mathrm{e}^{-x_{i}^{2}} \prod_{1 \leq i<j \leq n}\left|x_{i}-x_{j}\right|^{2 \gamma} \mathrm{d} x_{1} \cdots \mathrm{d} x_{n}
$$

and

$$
\langle f, g\rangle_{\alpha, \gamma}:=\int_{[0, \infty)^{n}} f(x) g(x) \prod_{i=1}^{n} x_{i}^{\alpha} \mathrm{e}^{-x_{i}} \prod_{1 \leq i<j \leq n}\left|x_{i}-x_{j}\right|^{2 \gamma} \mathrm{d} x_{1} \cdots \mathrm{d} x_{n} .
$$


The corresponding families of orthogonal polynomials are referred to as the multivariable Hermite and Laguerre polynomials, respectively. In particular note that

$$
\langle 1,1\rangle_{\gamma}=2^{\left(\begin{array}{c}
n \\
2
\end{array}\right) \gamma} \pi^{n / 2} F_{n}(\gamma)
$$

with $F_{n}$ the Mehta integral (1.5), and

$$
\langle 1,1\rangle_{\alpha, \gamma}=n ! W_{n}(\alpha+1, \gamma)
$$

with $W_{n}$ given by (3.19).

All of the orthogonal polynomials mentioned above admit $q$-analogues. In the $q$-theory the role of the Selberg integral is played by Askey's $q$-Selberg integral (2.7) and generalizations thereof. In the case of the Jacobi polynomials these $q$-analogues are known as the multivariable big and little $q$-Jacobi polynomials, and they were introduced by J.V. Stokman [157]. Stokman [158] also showed how the big and little $q$-Jacobi polynomials arise as special limits of the Koornwinder polynomials [100]. The latter are multivariable analogues of the Askey-Wilson orthogonal polynomials 14] and may be viewed as the generalizations of the Macdonald polynomials to the root system $\mathrm{BC}_{n}$. The relevant inner product in this case is given by [37, 100,

$$
\langle f, g\rangle_{t, t_{1}, \ldots, t_{4}}:=\frac{1}{(2 \pi)^{n}} \int_{-\pi}^{\pi} \cdots \int_{-\pi}^{\pi} f\left(\mathrm{e}^{\mathrm{i} x}\right) g\left(\mathrm{e}^{\mathrm{i} x}\right) \Delta\left(\mathrm{e}^{\mathrm{i} x} ; t, t_{1}, \ldots, t_{4}\right) \mathrm{d} x_{1} \cdots \mathrm{d} x_{n}
$$

where $\exp (\mathrm{i} x)=\left(\exp \left(\mathrm{i} x_{1}\right), \ldots, \exp \left(\mathrm{i} x_{n}\right)\right), \Delta\left(x ; t, t_{1}, \ldots, t_{4}\right)$ is the weight function (2.10) of Gustafson's constant term identity, and $f$ and $g$ are $\mathrm{BC}_{n}$ symmetric functions. $\left(f(z)\right.$ is $\mathrm{BC}_{n}$ symmetric if $f(\exp (\mathrm{i} x))$ is symmetric under signed permutations of $x=\left(x_{1}, \ldots, x_{n}\right)$.) The evaluation of $\langle 1,1\rangle_{t, \ldots, t_{4}}$ is of course provided by the right-hand side of (2.9).

\section{RECENT AND CURRENT RESEARCH DIRECTIONS}

4.1. The case of $\gamma$ a positive integer. Two recent studies have identified special features of the Selberg integral for $\gamma$ a positive integer. The first of these is due to J.-G. Luque and J.-Y. Thibon [105, 106], and it exhibits a connection with a special class of hyperdeterminants. The second, due to Stanley [154], gives a probabilistic interpretation of Selberg's integral.

For a $k$ th-order tensor $A=\left[A_{i_{1} i_{2} \cdots i_{k}}\right]$ on an $n$-dimensional space (so that $1 \leq$ $i_{p} \leq n$ ), the hyperdeterminant has been defined by A. Cayley (see references in [105]) as

$$
\operatorname{det}_{k}(A):=\sum_{\sigma_{2}, \ldots, \sigma_{k} \in \mathfrak{S}_{n}} \epsilon\left(\sigma_{2}\right) \cdots \epsilon\left(\sigma_{k}\right) \prod_{i=1}^{n} A_{i, \sigma_{2}(i), \ldots, \sigma_{k}(i)},
$$

where $\epsilon(\sigma)$ denotes the signature of the permutation $\sigma$. For $k$ odd this vanishes while $k=2$ corresponds to the usual definition of a determinant.

For an arbitrary measure $\mu(x)$ it is easy to see by use of the Vandermonde determinant formula that in the so-called Hankel case

$$
A_{i_{1} i_{2} \cdots i_{k}}=\mu_{i_{1}+i_{2}+\cdots+i_{k}-k}, \quad \mu_{j}:=\int_{-\infty}^{\infty} x^{j} \mathrm{~d} \mu(x),
$$

the corresponding hyperdeterminant is equal to a multiple integral,

$$
\operatorname{det}_{2 k}(A)=\frac{1}{n !} \int \cdots \int \prod_{1 \leq i<j \leq n}\left(x_{i}-x_{j}\right)^{2 k} \mathrm{~d} \mu\left(x_{1}\right) \cdots \mathrm{d} \mu\left(x_{n}\right) .
$$


For $\mathrm{d} \mu(x)=x^{\alpha-1}(1-x)^{\beta-1} \mathrm{~d} x$ on $x \in[0,1]$ this is precisely the Selberg integral with $\gamma=k$ an integer.

The probabilistic interpretation of the Selberg integral given in [154] applies for $\alpha=\beta=1$ and $\gamma$ a positive integer. In fact, as communicated to us by Stanley [155], this same probabilistic interpretation, extended to $\alpha, \beta$ general nonnegative integers, is already implied by appropriately interpreting supplementary problem I.25 of [153]. Following [155], the setting is to choose labelled points independently and with uniform distribution from the interval $[0,1]$. Specifically, for each $1 \leq$ $p \leq n$ and $(i, j)$ such that $1 \leq i<j \leq n$, choose $\alpha-1$ points labelled $y_{p}, \beta-1$ points labelled $z_{p}, n$ points labelled $t$ and $2 \gamma$ points labelled $a_{i j}$. Let $t_{i}$ be the $i$ th smallest point labelled $t$. The probability that any one of the points labelled $y_{p}$ is to the left of $t_{i}$ is $1-t_{i}$; the probability that any one of the points labelled $a_{i j}$ is between $t_{i}$ and $t_{j}$ for $i<j$ is $t_{i}-t_{j}$. It follows immediately that the Selberg integral $S_{n}(\alpha, \beta, \gamma)$ is the probability, $P_{S}$ say, that all the points labelled $y_{p}$ are to the left of $t_{p}$, and all the points labelled $z_{p}$ are to the right of $t_{p}$, and all the points labelled $a_{i j}$ lie between $t_{i}$ and $t_{j}$. Note that this statement remains valid for $2 \gamma$ an odd integer. An equivalent formulation (the one given in [153]) is to regard the selection of the labelled points uniformly and independently from $[0,1]$ as a random rearrangement of the symbols themselves (i.e. the $t$ 's, $y_{p}$ 's, $z_{p}$ 's and $a_{i j}$ 's), in which case $P_{S}$ corresponds to the probability that the rearrangement complies with the prescribed rule.

4.2. Random matrix theory. In this section we discuss three examples of interplays between random matrix theory and the Selberg integral. The first of these emphasizes the links with the Selberg and Dixon-Anderson densities. The other two examples show the importance of the Selberg integral in the development of random matrix theory.

It has been noted in 61] that the computations of Dixon and Anderson can be interpreted as giving the density of zeros of the random rational function

$$
R_{n+1}(x):=\sum_{i=1}^{n+1} \frac{w_{i}}{a_{i}-x},
$$

where the $w_{i}$ are distributed according to the Dirichlet distribution, to be denoted $D_{n+1}\left[s_{1}, \ldots, s_{n+1}\right]$,

$$
\frac{\Gamma\left(s_{1}+\cdots+s_{n+1}\right)}{\Gamma\left(s_{1}\right) \cdots \Gamma\left(s_{n+1}\right)} \prod_{i=1}^{n+1} w_{i}^{s_{i}-1}
$$

with $w_{1}, \ldots, w_{n+1}>0$ such that $w_{1}+\cdots+w_{n+1}=1$.

Let $S_{n}(\alpha, \beta, \gamma ; t)$ denote the Selberg density, being the normalized integrand of the Selberg integral. Now, motivated by the above interpretation of the DixonAnderson integral, a family of random polynomials $A_{j}(x), 1 \leq j \leq n$ were defined in [61] such that the zeros of $A_{j}(x)$ have PDF $S_{j}\left(\alpha_{j}, \beta_{j}, \gamma ; t\right)$ with $\alpha_{j}:=$ $(n-j) \gamma+\alpha, \beta_{j}:=(n-j) \gamma+\beta$. Setting $A_{-1}(x):=0, A_{0}(x):=1$, and specifying that $\left(w_{0}^{(j)}, w_{1}^{(j)}, w_{2}^{(j)}\right)$ be distributed according to the Dirichlet distribution $D_{3}\left[\beta_{j},(j-1) \gamma, \alpha_{j}\right]$, the polynomials $A_{j}(x)$ are determined by the random threeterm recurrence

$$
A_{j}(x)=w_{2}^{(j)}(x-1) A_{j-1}(x)+w_{0}^{(j)} x A_{j-1}(x)+w_{1}^{(j)} x(x-1) A_{j-2}(x) .
$$


Let $\alpha \mapsto \gamma \alpha / 2+1, \beta \mapsto \gamma \beta / 2+1$, and let the integrand of the Selberg integral be written in the form $\exp (-2 \gamma U)$ so that

$$
U=-\frac{\alpha}{2} \sum_{i=1}^{n} \log t_{i}-\frac{\beta}{2} \sum_{i=1}^{n} \log \left|1-t_{i}\right|-\sum_{1 \leq i<j \leq n} \log \left|t_{i}-t_{j}\right| .
$$

Then in the limit $\gamma \rightarrow \infty$, the Selberg density crystallizes to the minimum of $U$ subject to the constraint that $0<t_{i}<1$ for each $t_{i}$. According to a classical result of Stieltjes (see, e.g., [6]) this minimum is unique and occurs at the zeros of the Jacobi polynomial $P_{n}^{(\alpha-1, \beta-1)}(x)$. Indeed in the same limit the three term recurrence (4.1) is no longer random, and has solution $A_{j}(x)=\tilde{P}_{j}^{(n-j+\alpha-1, n-j+\beta-1)}(x)$ with $\tilde{P}_{j}^{(a, b)}(x)$ the monic form of the Jacobi polynomials.

The change of variables and limiting procedure giving rise to (1.8) reduces the Selberg density to the PDF (1.4). The Dixon-Anderson density permits a similar limit, and applied with $n \mapsto n+1, a_{0}=0$ and $a_{1}=1$ this results in the PDF on $\left\{t_{j}\right\}_{j=1}^{n+1}$ given by

$$
\begin{aligned}
& \frac{1}{\sqrt{2 \pi} \Gamma\left(s_{1}\right) \cdots \Gamma\left(s_{n}\right)} \frac{\prod_{1 \leq i<j \leq n+1}\left(t_{i}-t_{j}\right)}{\prod_{1 \leq i<j \leq n}\left(a_{i}-a_{j}\right)^{s_{i}+s_{j}-1}} \\
& \times \prod_{i=1}^{n+1} \prod_{j=1}^{n}\left|t_{i}-a_{j}\right|^{s_{j}-1} \exp \left(-\frac{1}{2} \sum_{j=1}^{n+1} t_{j}^{2}+\frac{1}{2} \sum_{j=1}^{n} a_{j}^{2}\right)
\end{aligned}
$$

supported on

$$
t_{1}>a_{1}>t_{2}>\cdots>a_{n}>t_{n+1} .
$$

The corresponding limit of $R_{n+1}(x)$ gives the random rational function

$$
\tilde{R}_{n+1}(x):=x-\mu_{0}+\sum_{i=1}^{n} \frac{\mu_{i}}{a_{i}-x},
$$

where $\mu_{0}$ has distribution $\mathrm{N}[0,1]$ and $\mu_{i}$ the Gamma distribution $\Gamma\left[s_{i}, 1\right]$. Indeed, the fact that the zeros of (4.3) have PDF (4.2) can readily be checked directly by adopting the strategy of Dixon and Anderson; see [52, 59]. Finally, the limiting form of the three term recurrence (4.1) is

$$
C_{j}(x)=(x-r) C_{j-1}(x)-s^{(j-1)} C_{j-2}(x)
$$

with $C_{-1}(x)=0, C_{0}(x)=1, r$ having distribution $\mathrm{N}[0,1]$ and $s^{(j)}$ distribution $\Gamma[j \gamma, 1]$. The random polynomial $C_{j}(x)$ has as the PDF for its zeros the density (1.4) with $n=j$ and $\beta=2 \gamma$.

It should be remarked that since (4.2) integrated over $t_{1}, \ldots, t_{n+1}$ gives unity, a limiting form of the Dixon-Anderson integral follows. Evans [52] used this, together with the strategy of Anderson, to give the first proof of the Mehta integral evaluation (1.6) which is independent of the Selberg integral.

The random polynomial $C_{n+1}(x)$ can be interpreted as the characteristic polynomial for a family of random matrices defined inductively [61] by

$$
M_{n+1}=\left[\begin{array}{cccc}
\lambda_{1}^{(n)} & & & b_{1} \\
& \ddots & & \vdots \\
& & \lambda_{n}^{(n)} & b_{n} \\
b_{1} & \ldots & b_{n} & c
\end{array}\right] .
$$


Here the $\lambda_{i}^{(n)}$ are the eigenvalues of $M_{n}, c$ has distribution $\mathrm{N}[0,1]$ and $b_{j}^{2}$ has distribution $\Gamma[\beta / 2,1]$. Indeed, it is straightforward to show that the eigenvalues of (4.5) are given by the zeros of (4.3), with $\mu_{0}=c, \mu_{i}=b_{i}$ and $a_{i}=\lambda_{i}^{(n)}$. In the case $\beta=1$, the invariance of members of the GOE with respect to conjugation by orthogonal matrices shows that (4.5) is similar to GOE matrices, and an analogous understanding of the relationship between (4.5) in the case $\beta=2$ and GUE matrices can be given. Moreover, it is generally true that a three-term recurrence

$$
p_{j}(x)=\left(x-a_{j}\right) p_{j-1}(x)-b_{j-1} p_{j-2}(x)
$$

with $p_{-1}(x):=0, p_{0}(x)=1$ is satisfied by the characteristic polynomial for the tridiagonal matrix

$$
\left[\begin{array}{ccccc}
a_{n} & b_{n-1} & & & \\
b_{n-1} & a_{n-1} & b_{n-2} & & \\
& \ddots & \ddots & \ddots & \\
& & b_{2} & a_{2} & b_{1} \\
& & & b_{1} & a_{1}
\end{array}\right]
$$

Hence $C_{n}(x)$ is also the characteristic polynomial for the above random tridiagonal matrix with each $a_{j}$ having distribution $\mathrm{N}[0,1]$ and with $b_{j}^{2}$ distributed as in (4.5). This is a result due to I. Dumitriu and A. Edelman [42], obtained without the use of (4.2). In this regard it should be mentioned that R. Killip and I. Nenciu 98], in a study which does not make use of the Dixon-Anderson integral (2.2), give the explicit construction of a family of random orthogonal matrices with eigenvalue $\mathrm{PDF}$ equal to the $\mathrm{BC}_{n}$ Selberg density, which itself is proportional to the integrand in (1.23). The methods of [42] and [98], which at a technical level proceed via a change of variables from a general tridiagonal matrix and unitary Hessenberg matrix to their eigenvalue/eigenvector decomposition, also yield the evaluations of the Mehta and Selberg integrals, respectively.

The second of our examples is a study by Forrester and Rains [62] focusing on the family of multi-dimensional integrals

$$
:=\int_{[0, x]^{p}} \int_{[x, 1]^{n-p}} \prod_{i=1}^{n} t_{i}^{\alpha-1}\left(1-t_{i}\right)^{\beta-1}\left|x-t_{i}\right|^{\tau-1} \prod_{1 \leq i<j \leq n}\left|t_{i}-t_{j}\right|^{2 \gamma} \mathrm{d} t_{1} \cdots \mathrm{d} t_{n}
$$

for $0 \leq p \leq n$ and $x \in[0,1]$. In the case $\tau=1$, the integral (4.6) is proportional to the probability that for the Selberg density, interpreted as an eigenvalue PDF, there are $p$ eigenvalues in the interval $[0, x]$ and $n-p$ eigenvalues in the interval $[x, 1]$; for $\tau=1+2 \gamma$ the integral (4.6) relates to the derivative of this quantity. The case $\tau=2 \gamma=1$ of this was first studied in the mathematical statistics literature for its relevance to canonical correlation analysis [35].

Theory connecting $I_{n, p}$ to a certain Fuchsian differential equation [35, 57, 117] implies that the integral is expressible as a linear combination of Frobenius solutions. These are solutions to the differential equation of the form

$$
g_{i}(x)=x^{\sigma_{i}} \sum_{k=0}^{\infty} a_{i, k} x^{k}, \quad \sigma_{i}=i(\alpha-1+\tau+(i-1) \gamma)
$$


for $0 \leq i \leq n$, and are normalized such that

$$
\lim _{x \rightarrow 0} \frac{g_{i}(x)}{I_{n, p}(x)}=1 \quad \text { for } \quad \operatorname{Re}\left(\sigma_{i}\right)>0 .
$$

A basic task - essentially equivalent to finding the monodromy matrix for the basis of integral solutions of the matrix Fuchsian system, of which (4.6) forms the top row - is to give the explicit form of the coefficients $c_{p, i}$ in the expansion

$$
I_{n, p}(x)=\sum_{i=0}^{n} c_{p, i} g_{i}(x) .
$$

One approach to this problem is to seek a regime in parameter space such that for $x \rightarrow 0$ the leading behaviour of $I_{n, p}(x)$ is proportional to $x^{\sigma_{i}}$. This is achieved by changing variables $t_{j}=x u_{j}$ for $1 \leq j \leq i$ where $p+1 \leq i \leq n$. A simple scaling of the integrand then shows that

$$
I_{n, p}(x) \sim x^{\sigma_{i}} S_{n-i}(\alpha+\tau+2 \gamma-1, \beta, \gamma) S_{i, p}(\alpha, \tau, \gamma),
$$

where $S_{n-i}$ is the Selberg integral and $S_{i, p}$ is the Dotsenko-Fateev integral (2.4). Recalling the normalization (4.7) and the recurrence (2.6) allows the sought coefficients to be calculated as

$$
\begin{aligned}
& c_{p, i}=0, \quad 0 \leq i \leq p-1, \\
& c_{p, i}=(-1)^{i-p} \prod_{j=1}^{i-p} \frac{\sin \pi(i-j+1) \gamma \sin \pi(\alpha+(i-j) \gamma)}{\sin \pi j \gamma \sin \pi(\alpha+\tau-1+(2 i-j-1) \gamma)}, \quad p \leq i \leq n,
\end{aligned}
$$

thus solving the problem at hand. Mimachi, using a different strategy, has recently obtained the same result [118].

Our final application stems from a question posed by B. Virág at a recent AMSIMS-SIAM summer research conference [1]. By way of background to his question, let us recall a result of Mehta and Dyson [116] which gives the circular ensembles identity alt $\left(\mathrm{COE}_{2 n}\right)=\mathrm{CSE}_{n}$. Here alt is the operation of integrating out every second eigenvalue, and the subscripts on the names of the ensembles indicate the total number of eigenvalues. Let us more generally introduce the notation $\mathrm{CE}_{\beta, n}$ for the PDF (1.9). The question posed by Virág was to investigate extensions of the result of Mehta and Dyson, in which blocks of eigenvalues in $\mathrm{CE}_{\beta, n}$ are integrated out to obtain another circular ensemble $\mathrm{CE}_{\beta^{\prime}, n^{\prime}}$. The Selberg integral is relevant for this purpose.

Let $p\left(k ; s ; \mathrm{CE}_{\beta, n}\right)$ denote the PDF for the spacing between eigenvalues which are $(k+1)$-st neighbours in the ensemble $\mathrm{CE}_{\beta, N}$. Let alt ${ }_{m}\left(\mathrm{CE}_{\beta, n}\right)$ denote the joint marginal distribution of every $m$ th eigenvalue in $\mathrm{CE}_{\beta, n}$. With this notation, if it were true that

$$
\operatorname{alt}_{m}\left(\mathrm{CE}_{\beta, m n}\right)=\mathrm{CE}_{\beta^{\prime}, n}
$$

for some $m, \beta, \beta^{\prime}$, then

$$
p\left(m k+m-1 ; s ; \mathrm{CE}_{\beta, m n}\right)=p\left(k ; s ; \mathrm{CE}_{\beta^{\prime}, n}\right) .
$$

Now the $k$-point correlation $\rho_{k}$ is obtained from (1.9) by integrating out the variables $\theta_{k+1}, \ldots, \theta_{n}$ and multiplying by $n ! /(n-k)$ !. It follows from this definition that $\rho_{k}$ is related to the small $s$ expansion of $p$ according to

$$
p\left(k ; s ; \mathrm{CE}_{\beta, n}\right) \sim \frac{2 \pi}{n k !} \int_{0}^{s} \cdots \int_{0}^{s} \rho_{k+2}\left(0, s, \theta_{1}, \ldots, \theta_{k}\right) \mathrm{d} \theta_{1} \cdots \mathrm{d} \theta_{k} .
$$


But for $\theta_{1}, \ldots, \theta_{n}$ small the definition of $\rho_{k}$ also implies that

$$
\rho_{k}\left(\theta_{1}, \ldots, \theta_{k}\right) \sim \frac{1}{(2 \pi)^{k}} \frac{n !}{(n-k) !} \frac{M_{n-k}(k \beta / 2, k \beta / 2, \beta / 2)}{M_{n}(0,0, \beta / 2)} \prod_{1 \leq i<j \leq k}\left|\theta_{i}-\theta_{j}\right|^{\beta},
$$

where $M_{n}$ refers to the Morris integral (1.17). Substituting (4.12) in (4.11) and scaling the integrand, an example of the Selberg integral is obtained, giving the formula

$$
\begin{aligned}
p\left(k ; s ; \mathrm{CE}_{\beta, n}\right) & \sim \frac{1}{(2 \pi)^{k+1}} \frac{(n-1) !}{k !(n-k-2) !} s^{k+\beta(k+2)(k+1) / 2} \\
& \times \frac{M_{n-k-2}((k+2) \beta / 2,(k+2) \beta / 2, \beta / 2)}{M_{n}(0,0, \beta / 2)} S_{k}(\beta+1, \beta+1, \beta / 2) .
\end{aligned}
$$

Using the gamma function evaluations (1.1) and (1.18), together with the duplication formula for the gamma function, one can check that in the case $m=r+1$, $\beta=2 /(r+1)$ and $\beta^{\prime}=2(r+1)$, (4.10) is compatible with (4.13). Thus, this investigation based on the Selberg integral reveals parameters for which the validity of (4.9) may be expected. One can in fact proceed further and prove, using a generalization of the Dixon-Anderson integral, that for these parameters (4.9) is indeed valid [60].

4.3. KZ equations and the Mukhin-Varchenko conjecture. On pages 510511 we saw that hypergeometric integrals of Selberg type arise naturally as solutions of (systems) of partial differential equations. There is a well-developed theory extending this to the setting of partial differential equations based on Lie algebras [46, 124, 143, 165]. These equations first arose in the context of conformal field theory, and are referred to as Knizhnik-Zamolodchikov (KZ) equations [99].

Let $\mathfrak{g}$ be a simple Lie algebra of rank $n$, with simple roots, fundamental weights and Chevalley generators given by $\alpha_{i}, \Lambda_{i}$ and $e_{i}, f_{i}, h_{i}$ for $1 \leq i \leq n$. Let $V_{\lambda}$ and $V_{\mu}$ be highest weight representations of $\mathfrak{g}$ with highest weights $\lambda$ and $\mu$, and let $u=u(z, w)$ be a function taking values in $V_{\lambda} \otimes V_{\mu}$ solving the KZ equation

$$
\kappa \frac{\partial u}{\partial z}=\frac{\Omega}{z-w} u, \quad \kappa \frac{\partial u}{\partial w}=\frac{\Omega}{w-z} u,
$$

where $\Omega \in \mathfrak{g} \otimes \mathfrak{g}$ is the Casimir element. (For the sake of simplicity we only consider the KZ equation in two variables, $z$ and $w$; for the more general case of $p$ variables $z_{1}, \ldots, z_{p}$, see, e.g., [165].) Let $\operatorname{Sing}_{\lambda, \mu}[\nu]$ denote the space of singular vectors of weight $\nu$ in $V_{\lambda} \otimes V_{\mu}$

$$
\operatorname{Sing}_{\lambda, \mu}[\nu]:=\left\{v \in V_{\lambda} \otimes V_{\mu}: h_{i} v=\nu\left(h_{i}\right) v, e_{i} v=0,1 \leq i \leq n\right\} .
$$

Then, according to a theorem of V.V. Schechtman and Varchenko [143], solutions $u$ with values in $\operatorname{Sing}_{\lambda, \mu}\left[\lambda+\mu-\sum_{i=1}^{n} k_{i} \alpha_{i}\right]$ are expressible in terms of multiple hypergeometric integrals

$$
u(z, w)=\sum u_{I J}(z, w) f^{I} v_{\lambda} \otimes f^{J} v_{\mu}
$$

with coordinate functions $u_{I J}$ given by

$$
u_{I J}(z, w)=\int_{\gamma} \Phi^{1 / \kappa}(z, w ; t) A_{I J}(z, w ; t) \mathrm{d} t_{1} \cdots \mathrm{d} t_{k} .
$$

Here $k:=k_{1}+\cdots+k_{n}, t:=\left(t_{1}, \ldots, t_{k}\right)$, the sum is over all ordered multisets $I$ and $J$ with elements taken from $\{1, \ldots, n\}$ such that their union contains the 
number $i$ exactly $k_{i}$ times, $v_{\lambda}$ and $v_{\mu}$ are the highest weight vectors of $V_{\lambda}$ and $V_{\mu}$, $f^{I} v:=\left(\prod_{i \in I} f_{i}\right) v$ and $\gamma$ is a suitable integration domain.

The functions $\Phi$ and $A_{I J}$ in the integrand of $u_{I J}$ are explicitly known. $A_{I J}$ is a rational function whose general form is too involved to explicitly state here (an example will be given below), and the function $\Phi$, known as the master function, is defined as follows. The first $k_{1}$ integration variables are attached to the simple root $\alpha_{1}$, the next $k_{2}$ integration variables are attached to the simple root $\alpha_{2}$, and so on, such that $\alpha_{t_{j}}:=\alpha_{i}$ if $k_{1}+\cdots+k_{i-1}<j \leq k_{1}+\cdots+k_{i}$. With this understood,

$$
\begin{aligned}
\Phi(z, w ; t)=(z-w)^{(\lambda, \mu)} \prod_{i=1}^{k}\left(t_{i}-z\right)^{-\left(\lambda, \alpha_{t_{i}}\right)}\left(t_{i}-w\right)^{-\left(\mu, \alpha_{t_{i}}\right)} & \times \prod_{1 \leq i<j \leq k}\left(t_{i}-t_{j}\right)^{\left(\alpha_{t_{i}}, \alpha_{t_{j}}\right)},
\end{aligned}
$$

where $($,$) is the bilinear symmetric form on \mathfrak{h}^{*}$ (the dual of the Cartan subalgebra $\mathfrak{h})$ normalized such that $(\theta, \theta)=2$ for the maximal root $\theta$.

The simplest possible example of a KZ solution contained in the SchechtmanVarchenko theorem corresponds to the rank 1 Lie algebra $\mathfrak{g}=\mathfrak{s l}_{2}=\mathrm{A}_{1}$, with simple root $\alpha_{1}$ and fundamental weight $\Lambda_{1}=\alpha_{1} / 2$. Taking $\lambda=m_{1} \Lambda_{1}$ and $\mu=$ $m_{2} \Lambda_{1}$ it follows that $u(z, w)$ takes values in the space of singular vectors of weight $\left(m_{1}+m_{2}-2 k_{1}\right) \Lambda_{1}$. Since $n=1$, it follows that $I=\left\{1^{r}\right\}$ and $J=\left\{1^{k_{1}-r}\right\}$ with $1 \leq r \leq k_{1}$, so that $u_{I J}, A_{I J}$ and $f^{I}$ can simply be denoted by $u_{r}, A_{r}$ and $f^{r}$. (In the case of rank one, there is no need for the index in $f_{1}$ ). Using $n$ instead of $k_{1}$ (so that $n$ no longer denotes the rank of the Lie algebra) and writing $v_{1}$ and $v_{2}$ instead of $v_{\lambda}=v_{m_{1} \Lambda_{1}}$ and $v_{\mu}=v_{m_{2} \Lambda_{2}}$, one finds, upon the assumption that $z<w$ are both real, that

$$
u(z, w)=\sum_{r=0}^{n} u_{r}(z, w) f^{r} v_{1} \otimes f^{n-r} v_{2}
$$

with

$$
\begin{aligned}
u_{r}(z, w)=(z-w)^{m_{1} m_{2} /(2 \kappa)} \int_{\gamma} A_{r}(z, w ; t) & \prod_{i=1}^{n}\left(t_{i}-z\right)^{-m_{1} / \kappa}\left(t_{i}-w\right)^{-m_{2} / \kappa} \\
& \times \prod_{1 \leq i<j \leq n}\left(t_{i}-t_{j}\right)^{2 / \kappa} \mathrm{d} t_{1} \cdots \mathrm{d} t_{n} .
\end{aligned}
$$

Here the domain of integration is the simplex $\gamma=\left\{t \in \mathbb{R}^{n} \mid z \leq t_{n} \leq \cdots \leq t_{1} \leq w\right\}$, and the rational function $A_{r}(z, w ; t)$ is given by

$$
A_{r}(z, w ; t)=\sum_{\substack{I \subseteq\{1, \ldots, n\} \\|I|=r}} \prod_{i \in I} \frac{1}{t_{i}-z} \prod_{i \notin I} \frac{1}{t_{i}-w}
$$

The coordinate functions $u_{r}$ are easily recognized as generalizations of the Selberg integral. In fact, for the extremal cases $r=0$ and $r=n$ they are exactly the 
Selberg integral. When $r=0$, for instance,

$$
\begin{aligned}
u_{0}(z, w)=(z-w)^{m_{1} m_{2} /(2 \kappa)} \int_{\gamma} \prod_{i=1}^{n}\left(t_{i}-z\right)^{-m_{1} / \kappa}\left(t_{i}-w\right)^{-m_{2} / \kappa-1} & \\
& \times \prod_{1 \leq i<j \leq n}\left(t_{i}-t_{j}\right)^{2 / \kappa} \mathrm{d} t_{1} \cdots \mathrm{d} t_{n} .
\end{aligned}
$$

Making the change of variables $t_{i}=(w-z) s_{i}+z$ for $1 \leq i \leq n$, this yields

$$
u_{0}(z, w)=\frac{(-1)^{A}(z-w)^{B}}{n !} S_{n}\left(1-\frac{m_{1}}{\kappa},-\frac{m_{2}}{\kappa}, \frac{1}{\kappa}\right),
$$

where $A=n\left(n-1-m_{1}\right) / \kappa+n$ and $B=\left(m_{1} m_{2}-2 n\left(m_{1}+m_{2}\right)+2 n(n-1)\right) /(2 \kappa)$.

In 2000 E. Mukhin and Varchenko [124] formulated a surprising conjecture regarding the scaled master function

$$
\Phi(t)=\prod_{i=1}^{k} t_{i}^{-\left(\lambda, \alpha_{t_{i}}\right)}\left(1-t_{i}\right)^{-\left(\mu, \alpha_{t_{i}}\right)} \prod_{1 \leq i<j \leq n}\left(t_{i}-t_{j}\right)^{\left(\alpha_{t_{i}}, \alpha_{t_{j}}\right)} .
$$

They conjectured that if the space $\operatorname{Sing}\left[\lambda+\mu-\sum_{i=1}^{n} k_{i} \alpha_{i}\right]$ of singular vectors is one-dimensional, then

$$
\int|\Phi(t)|^{1 / \kappa} \mathrm{d} t_{1} \cdots \mathrm{d} t_{k}
$$

is expressible as a product of gamma functions. Neither the exact integration domain nor the specific form for the product of gamma functions is contained in the Mukhin-Varchenko conjecture.

For $\mathfrak{g}=\mathfrak{s l}_{2}=\mathrm{A}_{1}$, the conjecture corresponds to the evaluation of the Selberg integral. For $\mathfrak{g}=\mathfrak{s l}_{n+1}=\mathrm{A}_{n}$, and $\operatorname{Sing}_{\lambda, \mu}\left[\lambda+\mu-\sum_{i=1}^{n} \alpha_{i}\right]$ with $\lambda=\Lambda_{1}, \mu=$ $\sum_{i=1}^{n} \mu_{i} \Lambda_{i}$, the conjecture simply follows by iterating the beta integral (1.3); see [124]. For $\mathfrak{g}=\mathrm{B}_{n}, \mathrm{C}_{n}$ or $\mathrm{D}_{n}$ and

$$
\operatorname{Sing}_{\Lambda_{1}, \Lambda_{1}}\left[2 \Lambda_{1}-r \alpha_{n-1}-s \alpha_{n}-2 \sum_{i=1}^{n-2} \alpha_{i}\right] \quad \text { with } \quad(r, s)= \begin{cases}(2,2) & \mathrm{B}_{n}, \\ (2,1) & \mathrm{C}_{n} \\ (1,1) & \mathrm{D}_{n}\end{cases}
$$

(corresponding to the tensor product of the vector representation of $\mathfrak{g}$ ), Mimachi and T. Takamuki [121] established the Mukhin-Varchenko conjecture by iterating the Selberg integral for $n=2$ ( $\mathrm{B}_{n}$ case) or the beta integral $\left(\mathrm{C}_{n}\right.$ and $\mathrm{D}_{n}$ cases).

In $2003 \mathrm{~V}$. Tarasov and Varchenko [162] employed KZ equations and the closely related dynamical equations to settle the conjecture for $\mathfrak{g}=\mathfrak{s l}_{3}=\mathrm{A}_{2}$. In recent work by Warnaar [168, 169, 170], an approach to the $\mathfrak{s l}_{n+1}=\mathrm{A}_{n}$ case of the Mukhin-Varchenko conjecture was developed, based on the theory of Macdonald polynomials and generalized hypergeometric series. Specifically, the integral (4.14) for $\mathfrak{g}=\mathrm{A}_{n}$ can be evaluated in closed form when $\lambda=\lambda_{n} \Lambda_{n}$ and $\mu=\sum_{i} \mu_{i} \Lambda_{i}$ (or when $\lambda=\lambda_{1} \Lambda_{1}$ and $\mu=\sum_{i} \mu_{i} \Lambda_{i}$ ). Stripping the integral from its Lie algebra notation and using $\alpha_{i}$ and $\beta_{i}(1 \leq i \leq n)$ for exponents in the integral (so that the $\alpha_{i}$ no longer denote the simple roots) the $\mathrm{A}_{n}$ Selberg integral can be stated 
explicitly as

$$
\begin{aligned}
& \int \prod_{s=1}^{n}\left[\left|\Delta\left(t^{(s)}\right)\right|^{2 \gamma} \prod_{i=1}^{k_{s}}\left(t_{i}^{(s)}\right)^{\alpha_{s}-1}\left(1-t_{i}^{(s)}\right)^{\beta_{s}-1}\right] \prod_{s=1}^{n-1}\left|\Delta\left(t^{(s)}, t^{(s+1)}\right)\right|^{-\gamma} \mathrm{d} t \\
& =\prod_{1 \leq s \leq r \leq n} \prod_{i=1}^{k_{s}-k_{s-1}} \frac{\Gamma\left(\beta_{s}+\cdots+\beta_{r}+(i+s-r-1) \gamma\right)}{\Gamma\left(\alpha_{r}+\beta_{s}+\cdots+\beta_{r}+\left(i+s-r+k_{r}-k_{r+1}-2\right) \gamma\right)} \\
& \quad \times \prod_{s=1}^{n} \prod_{i=1}^{k_{s}} \frac{\Gamma\left(\alpha_{s}+\left(i-k_{s+1}-1\right) \gamma\right) \Gamma(i \gamma)}{\Gamma(\gamma)} .
\end{aligned}
$$

Here $k_{1}, \ldots, k_{n+1}$ are nonnegative integers such that $k_{n+1}=0$ and $k_{1} \leq k_{2} \leq \cdots \leq$ $k_{n}$, the exponents $\alpha_{1}, \ldots, \alpha_{n}, \beta_{1}, \ldots, \beta_{n}, \gamma \in \mathbb{C}$ such that $\alpha_{1}=\cdots=\alpha_{n-1}=1$ and such that both sides of the identity are well defined. Furthermore, $t^{(s)}=$ $\left(t_{1}^{(s)}, \ldots, t_{k_{s}}^{(s)}\right)$ is the set of variables attached to the $s$ th simple root of $\mathrm{A}_{n}$,

$$
\Delta(u)=\prod_{1 \leq i<j \leq l_{u}}\left(u_{i}-u_{j}\right) \quad \text { and } \quad \Delta(u, v)=\prod_{i=1}^{l_{u}} \prod_{j=1}^{l_{v}}\left(u_{i}-v_{j}\right)
$$

for sets of variables $u=\left(u_{1}, \ldots, u_{l_{u}}\right)$ and $v=\left(v_{1}, \ldots, v_{l_{v}}\right)$, and $\mathrm{d} t=\mathrm{d} t^{(1)} \cdots \mathrm{d} t^{(n)}$ with $\mathrm{d} t^{(s)}=\mathrm{d} t_{1}^{(s)} \cdots \mathrm{d} t_{k_{s}}^{(s)}$ so that the integral is $\left(k_{1}+\cdots+k_{n}\right)$-dimensional.

Not yet specified in the $A_{n}$ Selberg integral is the domain of integration, which, unfortunately, is rather involved. A key ingredient is the set of maps

$$
M_{s}:\left\{1, \ldots, k_{s}\right\} \rightarrow\left\{1, \ldots, k_{s+1}\right\}
$$

such that

$$
M_{s}(i) \leq M_{s}(i+1) \quad \text { and } \quad 1 \leq M_{s}(i) \leq k_{s+1}-k_{s}+i .
$$

A standard counting argument shows that there are exactly $c_{k_{s+1}, k_{s}}$ admissible $M_{s}$, where $c_{n, k}$ is the row $(n, k)$ entry in the Catalan triangle, or, equivalently, the number of standard Young tableaux of shape $(n, k)$. Given $M_{s}$, fix an ordering among the $t_{i}^{(s)}$ and $t_{j}^{(s+1)}$ as

$$
t_{M_{s}(i)}^{(s+1)} \leq t_{i}^{(s)} \leq t_{M_{s}(i)-1}^{(s+1)} \quad \text { for } 1 \leq i \leq k_{s},
$$

where $t_{0}^{(s+1)}:=\infty$. Given admissible maps $M_{1}, \ldots, M_{n-1}$, define $D_{M_{1}, \ldots, M_{n-1}}^{k_{1}, \ldots, k_{n}}$ as the set of points

$$
\left(t_{1}^{(1)}, \ldots, t_{k_{1}}^{(1)}, t_{1}^{(2)}, \ldots, t_{k_{2}}^{(2)}, \ldots, t_{1}^{(n)}, \ldots, t_{k_{n}}^{(n)}\right)
$$

such that (4.15) holds for all $1 \leq s \leq n-1$ and

$$
0 \leq t_{k_{s}}^{(s)} \leq \cdots \leq t_{1}^{(s)} \leq 1
$$

holds for all $1 \leq s \leq n$. Then the domain of integration, written as a chain, is given by

$$
\sum_{M_{1}, \ldots, M_{n-1}} F_{M_{1}, \ldots, M_{n-1}}^{k_{1}, \ldots, k_{n}}(\gamma) D_{M_{1}, \ldots, M_{n-1}}^{k_{1}, \ldots, k_{n}}
$$

where

$$
F_{M_{1}, \ldots, M_{n-1}}^{k_{1}, \ldots, k_{n}}(\gamma)=\prod_{s=1}^{n-1} \prod_{i=1}^{k_{s}} \frac{\sin \left(\pi\left(i+k_{s+1}-k_{s}-M_{s}(i)+1\right) \gamma\right)}{\sin \left(\pi\left(i+k_{s+1}-k_{s}\right) \gamma\right)}
$$


In complete analogy with the ordinary Selberg integral, the evaluation of the $\mathrm{A}_{n}$ Selberg integral can be generalized to include a Jack polynomial in the integrand, thus generalizing the Kadell integral (3.13), see [169].

4.4. Elliptic Selberg integrals. In the last few years there has been rapid progress in the field of elliptic generalizations of hypergeometric series; see [67, 151]. Classical hypergeometric series $\sum_{n=0}^{\infty} c_{n}$ are characterized by the ratio $c_{n+1} / c_{n}$ being a rational function of $n$. Their elliptic counterparts have the same ratio equal to an elliptic function of $n$.

The classical hypergeometric series permits elliptic generalizations, as do related integrals, such as the Euler beta integral (1.3). In the elliptic theory the ordinary gamma function must be replaced by what is known as the elliptic gamma function,

$$
\Gamma(z ; p, q)=\prod_{i, j=0}^{\infty} \frac{1-z^{-1} p^{i+1} q^{j+1}}{1-z p^{i} q^{j}},
$$

defined for $|p|,|q|<1$. This function can be traced back to E.W. Barnes in 1904 [18], but was given prominence through the recent work of S.N.M. Ruijsenaars [142]. It permits the extension of the standard gamma recurrence to

$$
\Gamma(q z ; p, q)=\theta(z ; p) \Gamma(z ; p, q),
$$

where $\theta(z ; p)=(z ; p)_{\infty}(p / z ; p)_{\infty}$ is a normalized theta function. Another fundamental property of the elliptic gamma function is the functional equation

$$
\Gamma(z ; p, q)=\frac{1}{\Gamma(p q / z ; p, q)},
$$

which follows immediately from definition (4.16).

The elliptic analogue of the beta integral (1.3) was discovered in 2000 by V.P. Spiridonov [148],

$$
\int_{\mathbb{T}} \frac{\prod_{r=1}^{6} \Gamma\left(t_{r} z^{ \pm 1} ; p, q\right)}{\Gamma\left(z^{ \pm 2} ; p, q\right)} \frac{\mathrm{d} z}{2 \pi \mathrm{i} z}=\frac{2}{(p ; p)_{\infty}(q ; q)_{\infty}} \prod_{1 \leq r<s \leq 6} \Gamma\left(t_{r} t_{s} ; p, q\right),
$$

where each $\left|t_{r}\right|<1, \mathbb{T}$ is the positively oriented unit circle, $\prod_{r=1}^{6} t_{r}=p q$ and

$$
\Gamma\left(t z^{ \pm m} ; p, q\right):=\Gamma\left(t z^{m} ; p, q\right) \Gamma\left(t z^{-m} ; p, q\right) .
$$

The $p \rightarrow 0$ limit is the well-known Rahman integral [107, 133], which itself is an extension of the Askey-Wilson integral [14]. For the reduction of this last integral to the beta integral (1.3), see [67].

J.F. van Diejen and Spiridonov [39] have given an $n$-dimensional generalization of (4.18), which may be viewed as an elliptic extension of the Selberg integral. This integral, the $p \rightarrow 0$ limit of which was first obtained by Gustafson [2], takes the form

$$
\begin{array}{r}
\int_{\mathbb{T}^{n}} \prod_{1 \leq i<j \leq n} \frac{\Gamma\left(t z_{i}^{ \pm 1} z_{j}^{ \pm 1} ; p, q\right)}{\Gamma\left(z_{i}^{ \pm 1} z_{j}^{ \pm 1} ; p, q\right)} \prod_{i=1}^{n} \frac{\prod_{r=1}^{6} \Gamma\left(t_{r} z_{i}^{ \pm 1} ; p, q\right)}{\Gamma\left(z_{i}^{ \pm 2} ; p, q\right)} \frac{\mathrm{d} z_{1}}{2 \pi \mathrm{i} z_{1}} \cdots \frac{\mathrm{d} z_{n}}{2 \pi \mathrm{i} z_{n}} \\
=\frac{2^{n} n !}{(p ; p)_{\infty}^{n}(q ; q)_{\infty}^{n}} \prod_{j=1}^{n}\left(\frac{\Gamma\left(t^{j} ; p, q\right)}{\Gamma(t ; p, q)} \prod_{1 \leq r<s \leq 6} \Gamma\left(t^{j-1} t_{r} t_{s} ; p, q\right)\right),
\end{array}
$$

where $|t|,\left|t_{1}\right|, \ldots,\left|t_{6}\right|<1$ and $t^{2 n-2} \prod_{r=1}^{6} t_{j}=p q$. van Diejen and Spiridonov provided a proof of (4.19) along the lines of the Anderson and Gustafson proofs of 
(1.1) and (2.9), respectively. This required an elliptic generalization of the DixonAnderson integral (2.2) which, initially, was proved making an assumption about the vanishing of certain elliptic integrals. The first complete proof of the elliptic Dixon-Anderson integral was found by Rains [134]. Later Spiridonov [150] and Rains and Spiridonov [137] found two more proofs of the same result.

The reduction of the elliptic Selberg integral (4.19) to the ordinary Selberg integral is rather cumbersome, requiring several limits, variables changes and specializations of parameters [136]. Fairly straightforward, however, is to see that (4.19) provides an elliptic extension of Gustafson's $\mathrm{BC}_{n}$ constant term identity (2.9). To see this, one first needs to eliminate $t_{6}$ using $t^{2 n-2} \prod_{r=1}^{6} t_{r}=p q$. This gives rise to several elliptic gamma functions of the form $\Gamma(p q A)$ which, by (4.17), may be replaced by $1 / \Gamma(1 / A)$. After these elementary manipulations, the $p \rightarrow 0$ limit can be carried out, using that $\Gamma(z ; 0, q)=1 /(z ; q)_{\infty}$. Finally, taking $t_{5}=0$ and interpreting the resulting integral as a constant term identity yields (2.9).

Analogous to (3.22), the integrand of (4.19) can be used to define an inner product. Rains [134] has specified a family of abelian functions which are biorthogonal with respect to this inner product, extending the Rahman-Spiridonov theory [133, 149] of such functions to the multivariable setting, as well as generalizing the Koornwinder polynomials and Okounkov $\mathrm{BC}_{n}$ interpolation polynomials [127] to the elliptic level. (The elliptic interpolation polynomials were independently introduced by H. Coskun and Gustafson in 34 without the use of elliptic Selberg type integrals.) Rains also extended the integrand of (4.19) analogous to the ${ }_{2} F_{1}$ extension (3.16) of the Selberg integral, and obtained transformation formulas for the resulting elliptic hypergeometric integrals. By considering the reduction of his theory to the Selberg level, Rains obtained [135], for example,

$$
\begin{array}{r}
\int_{0}^{1} \cdots \int_{0}^{1} P_{\lambda}^{(1 / \gamma)}(t) P_{\mu}^{(1 / \gamma)}(t) \prod_{i=1}^{n} t_{i}^{\alpha-1}\left(1-t_{i}\right)^{\gamma-1} \prod_{1 \leq i<j \leq n}\left|t_{i}-t_{j}\right|^{2 \gamma} \mathrm{d} t_{1} \cdots \mathrm{d} t_{n} \\
=\prod_{i, j=1}^{n} \frac{\Gamma\left(\alpha+(2 n-i-j)+\lambda_{i}+\mu_{j}\right)}{\Gamma\left(\alpha+(2 n-i-j+1)+\lambda_{i}+\mu_{j}\right)} \prod_{j=0}^{n-1} \frac{\Gamma((j+1) \gamma) \Gamma(1+(j+1) \gamma)}{\Gamma(1+\gamma)} \\
\times P_{\lambda}^{(1 / \gamma)}\left(1^{n}\right) P_{\mu}^{(1 / \gamma)}\left(1^{n}\right) .
\end{array}
$$

This integral, which generalizes the $\beta=\gamma$ case of Kadell's integral (3.13) is originally due to Kadell [86] and (for $\gamma=1$ ) L.K. Hua [78]. Kadell's integral (3.13) also has an elliptic analogue, which has the feature that the Dotsenko-Fateev integral (2.4) is a special case [135].

There are other integrals in the literature referred to as elliptic Selberg integrals, although they do not contain the actual Selberg integral as a limiting case. These integrals arise as solutions to the Knizhnik-Zamolodchikov-Bernard (KZB) heat equation for $(2 n+1)$-dimensional $\mathfrak{s l}_{2}$ modules

$$
2 \pi \mathrm{i} \kappa \frac{\partial u}{\partial \tau}=\frac{\partial^{2} u}{\partial \lambda^{2}}+n(n+1) \rho^{\prime}(\lambda, \tau) u
$$

Here $u=u(\lambda, \tau), \rho(\lambda, \tau)=\vartheta^{\prime}(\lambda, \tau) / \vartheta(\lambda, \tau)$ with differentiation with respect to $\lambda$, and $\vartheta(\lambda, \tau)=\theta_{1}(\pi \lambda, \tau)$ is a theta function [172]. 
To describe the relevant solutions to the KZB equations, let $\Phi$ be the elliptic master function

$$
\Phi\left(t_{1}, \ldots, t_{n} ; \tau\right)=\prod_{i=1}^{n} E\left(t_{i}, \tau\right)^{-2 n} \prod_{1 \leq i<j \leq n} E\left(t_{i}-t_{j}, \tau\right)^{2},
$$

where $E(t, \tau)$ is the elliptic analogue of $t$

$$
E(t, \tau)=\frac{\vartheta(t, \tau)}{\vartheta^{\prime}(0, \tau)}
$$

The solutions considered by G. Felder, L. Stevens and Varchenko [56] are the linear combinations

$$
u_{\kappa, m}(\lambda, \tau)=J_{\kappa, m}(\lambda, \tau)+(-1)^{n+1} J_{\kappa, m}(-\lambda, \tau),
$$

where

$$
J_{\kappa, m}(\lambda, \tau):=\int_{0<t_{n}<\cdots<t_{1}<1} \Phi^{1 / \kappa}\left(t_{1}, \ldots, t_{n} ; \tau\right) \theta_{\kappa, m}\left(\lambda+\frac{2|t|}{\kappa}, \tau\right) \prod_{i=1}^{n} \sigma_{\lambda}\left(t_{i}, \tau\right) \mathrm{d} t_{1} \cdots \mathrm{d} t_{n} .
$$

Whenever necessary, this integral is understood in the sense of analytic continuation from the region where the exponents in $\Phi^{1 / \kappa}$ have positive real part [56], $|t|=$ $t_{1}+\cdots+t_{n}, \theta_{\kappa, m}(t, \tau)$ is a theta function of degree $\kappa$ and characteristic $m$

$$
\theta_{\kappa, m}(\lambda, \tau)=\sum_{j \in \mathbb{Z}+\frac{m}{2 \kappa}} \mathrm{e}^{2 \pi \mathrm{i} \kappa(\tau j+\lambda) j}
$$

and $\sigma_{\lambda}(t, \tau)=\theta(\lambda-t, \tau) /(\theta(\lambda, \tau) E(t, \tau))$.

In several instances Felder, Stevens and Varchenko found that the "elliptic Selberg integrals" $u_{\kappa, m}(\lambda, \tau)$ permit closed form evaluations in terms of theta functions and ordinary gamma functions. The simplest case of such an evaluation corresponds to

$$
\begin{aligned}
u_{2 n+2, n+1}(\lambda, \tau)=(2 \pi)^{n / 2} \mathrm{e}^{-\pi \mathrm{i} \frac{n(3 n-1)}{4(n+1)}} \mathrm{e}^{\pi \mathrm{i} \frac{n+1}{2}} \theta(\lambda, \tau)^{n+1} & \\
& \times S_{n}\left(\frac{n+2}{2(n+1)},-\frac{n}{n+1}, \frac{1}{2(n+1)}\right) \prod_{i=1}^{n}\left(1-\mathrm{e}^{2 \pi \mathrm{i} \frac{n+1+i}{2(n+1)}}\right),
\end{aligned}
$$

where $S_{n}$ is the Selberg integral (1.11).

4.5. The value distributions of the Riemann $\zeta$ function on the critical line. The final topic to be reviewed, following J.P. Keating and N.C. Snaith [94], is the use of the Selberg integral in providing quantitive predictions for properties of the Riemann zeta function $\zeta(s)$ on the critical line $\operatorname{Re}(s)=1 / 2$. There are two such applications, one relating to the moments of $|\zeta(1 / 2+i t)|$, the other to the value distribution of $\log \zeta(1 / 2+\mathrm{i} t)$ for large $t$. We begin by reviewing the status of both problems prior to [94].

Interest in the averaged moments

$$
\frac{1}{T} \int_{0}^{T}|\zeta(1 / 2+\mathrm{i} t)|^{2 a} \mathrm{~d} t
$$

goes back to the work of Hardy and Littlewood [75], who studied the case $a=$ 1. Subsequent work indicates that (4.20) for general $a$ exhibits the leading form 
$(\log T)^{a^{2}} f(a) A(a)$. Here $A(a)$ is an explicitly known number theoretic quantity, while

$$
f(1)=1, \quad f(2)=\frac{1}{12}, \quad f(3)=\frac{42}{9 !} \quad \text { and } \quad f(4)=\frac{24024}{16 !} .
$$

The calculation of each of these values is increasingly complex, and an outstanding problem then is to compute $f(a)$ for general values of $a$.

In relation to the value distribution of $\log \zeta(1 / 2+\mathrm{i} t)$ for large $t$, a theorem of Selberg [146] asserts that for any rectangle $B \in \mathbb{C}$,

$$
\lim _{T \rightarrow \infty} \frac{1}{T}\left|\left\{t: T \leq t \leq 2 T, \frac{\log \zeta(1 / 2+\mathrm{i} t)}{\sqrt{\frac{1}{2} \log \log T}} \in B\right\}\right|=\frac{1}{2} \iint_{B} \mathrm{e}^{-\frac{1}{2}\left(x^{2}+y^{2}\right)} \mathrm{d} x \mathrm{~d} y
$$

In words this says that the real and complex parts of $\log \zeta(s)$ on the critical line are distributed as independent Gaussian random variables. On the other hand, this same distribution can be calculated numerically for large $\left(\approx 10^{19}\right)$ but finite values of $t$, using data from Odlyzko's high precision computer computation of large Riemann zeros [125]. Significant deviation from a Gaussian distribution is found; see, e.g., the plot in [96]. The problem is to isolate the mechanism responsible for the deviation, and use this to predict the shape of the distribution for general large but finite $t$.

Both problems were solved in 94]. The key conceptual idea comes by way of an hypothesis, the Keating-Snaith hypothesis, which is an extension of the Montgomery-Odlyzko law linking the Riemann zeros to eigenvalues of large complex Hermitian random matrices; see, e.g., 93].

Let $\Lambda(z)$ denote the characteristic polynomial of matrices from the CUE (the $\beta=2$ case of (1.9) ). Then the Keating-Snaith hypothesis asserts that the number theoretic independent factors in the value distribution of $|\zeta(1 / 2+i t)|$ and $\log \zeta(1 / 2+\mathrm{i} t)$ will for large $t$ coincide with the value distribution of $|\Lambda(z)|$ and $\log \Lambda(z)$ for $|z|=1$ and large matrix size $n$. The value of $n$ in the CUE is to be related to $t$ in $\zeta(1 / 2+\mathrm{i} t)$ by

$$
n=\log t
$$

which ensures that the density of eigenvalues and the density of Riemann zeros are the same to leading order.

To apply the hypothesis to the value distribution of $\log \zeta(1 / 2+\mathrm{i} t)$, note that by writing $\Lambda(z)=\prod_{i=1}^{n}\left(\exp \left(\mathrm{i} \theta_{i}\right)-z\right)$ it follows that

$$
\operatorname{Re} \log \Lambda(-1)=\sum_{i=1}^{n} \log \left|\mathrm{e}^{\mathrm{i} \theta_{i}}+1\right| \quad \text { and } \quad \operatorname{Im} \log \Lambda(-1)=\frac{1}{2} \sum_{i=1}^{n} \theta_{i} .
$$

Therefore, with $\delta(x)$ the Dirac delta function,

$$
\begin{array}{r}
\int_{-\infty}^{\infty} \int_{-\infty}^{\infty}\langle\delta(s-\operatorname{Re} \log \Lambda(-1)) \delta(t-\operatorname{Im} \log \Lambda(-1))\rangle_{\mathrm{CUE}} \mathrm{e}^{\mathrm{i}(l s+k t)} \mathrm{d} s \mathrm{~d} t \\
=\left\langle\prod_{i=1}^{n} \mathrm{e}^{\frac{1}{2} \mathrm{i} k \theta_{i}}\left|1+\mathrm{e}^{\mathrm{i} \theta_{i}}\right|^{\mathrm{i} l}\right\rangle_{\mathrm{CUE}}
\end{array}
$$

Now scale

$$
k \mapsto k\left(\frac{2}{\log n}\right)^{1 / 2} \quad \text { and } \quad l \mapsto l\left(\frac{2}{\log n}\right)^{1 / 2}
$$


which, by (4.23), corresponds to a scaling of $\log \zeta(1 / 2+\mathrm{i} t)$ by

$$
\left(\frac{1}{2} \log \log T\right)^{1 / 2} \text {. }
$$

Then the hypothesis predicts (4.24) to be the Fourier transform of the value distribution of $\log \zeta(1 / 2+\mathrm{i} t)$ scaled by (4.26). To compute the random matrix average, recall (1.9), and note that the average in (4.24) is essentially the Morris integral (1.17) for $\gamma=1$. By (1.18) this has the gamma function evaluation (1.18). Numerical inversion gives a prediction for the value distribution that agrees with the one obtained from Odlyzko's data. Furthermore, the scaled form of (4.24) with the substitutions (4.25) has been computed in [15] to be equal to $\exp \left(-\left(k^{2}+l^{2}\right) / 2\right)$. This shows that the Keating-Snaith hypothesis is in quantitive agreement with Selberg's theorem (4.22).

The moment of $|\Lambda(-1)|$ relevant to (4.20) is the average (4.24) with $k=0$, $\mathrm{i} l=2 a$, with the identification (4.23). The Keating-Snaith hypothesis predicts that

$$
f(a)=\lim _{n \rightarrow \infty} n^{-a^{2}}\left\langle\prod_{i=1}^{n}\left|1+\mathrm{e}^{\mathrm{i} \theta_{i}}\right|^{2 a}\right\rangle_{\mathrm{CUE}}=\frac{G^{2}(a+1)}{G(2 a+1)},
$$

$G(x)$ denoting the Barnes $G$-function, where the second equality follows upon an application of (1.18). For $a=1, \ldots, 4$, this correctly reproduces (4.21).

It should also be remarked that the value distribution of $\Lambda( \pm 1)$ for $\Lambda(z)$ the characteristic polynomial of a random orthogonal or unitary symplectic matrix, chosen with Haar measure, is a special case of (1.23), and thus similarly is an example of the Selberg integral. Keating and Snaith [95] make use of this fact to provide a quantitative link between the value distribution of families of $L$-functions on the critical line and random matrix theory.

\section{ACKNOWLEDGEMENTS}

We gratefully acknowledge helpful correspondence on the mathematics and history of the Selberg integral from George E. Andrews, Enrico Bombieri, Freeman J. Dyson, Ron J. Evans, Jon P. Keating, Eric M. Rains, Amitai Regev, Vyacheslav P. Spiridonov and Richard P. Stanley.

This work has been supported by the Australian Research Council.

\section{About THE AUthors}

Peter Forrester is a professor at the University of Melbourne and a member of the Australian Academy of Science. His book Log-Gases and Random Matrices, which covers several of the topics discussed in this paper in further detail, is soon to be published by Princeton University Press.

Ole Warnaar is a professor at the University of Queensland and vice president of the Australian Mathematical Society. He is a member of the Australian Academy of Science.

\section{REFERENCES}

[1] AMS-IMS-SIAM meeting Interactions of Random Matrix Theory, Integrable Systems, and Stochastic Processes, June 2007.

[2] G.W. Anderson, The evaluation of Selberg sums, C. R. Acad. Sci. Paris Sér. I Math. 311 (1990), 469-472. MR1076474 (91m:11109) 
[3] G.W. Anderson, A short proof of Selberg's generalized beta formula, Forum Math. 3 (1991), 415-417. MR1115956 (92j:33006)

[4] G.E. Andrews, Problems and prospects for basic hypergeometric functions, in Theory and Application of Special Functions, Math. Res. Center, Univ. Wisconsin, Publ. No. 35, Academic Press, New York, 1975; pp. 191-224. MR0399528 (53:3372)

[5] G. E. Andrews and R. Askey, Another q-extension of the beta function, Proc. Amer. Math. Soc. 81 (1981), 97-100. MR589145 (81j:33001)

[6] G. E. Andrews, R. Askey and R. Roy, Special functions, Encyclopedia of Mathematics and its Applications, Vol. 71, Cambridge University Press, Cambridge, 1999. MR 1688958 (2000g:33001)

[7] K. Aomoto, Jacobi polynomials associated with Selberg integrals, SIAM J. Math. Anal. 18 (1987), 545-549. MR876291 (88h:17016)

[8] K. Aomoto, The complex Selberg integral, Quart. J. Math. Oxford 38 (1987), 385-399. MR.916224 (89g:32004)

[9] K. Aomoto, On a theta product formula for the symmetric A-type connection function, Osaka J. Math. 32 (1995), 35-39. MR.1323100 (96d:33010)

[10] K. Aomoto, On elliptic product formulas for Jackson integrals associated with reduced root systems, J. Algebraic Combin. 8 (1998), 115-126. MR1648460(2000b:33012)

[11] R. Askey, Some basic hypergeometric extensions of integrals of Selberg and Andrews, SIAM J. Math. Anal. 11 (1980) 938-951. MR595822 (82e:33002)

[12] R. Askey, Letter to the SIAM minisymposium "Problems and solutions in special functions", in: OP-SF NET 5.5 (Web resource), 1998.

[13] R. Askey and D. Richards, Selberg's second beta integral and an integral of Mehta, in Probability, Statistics, and Mathematics: Papers in Honor of Samuel Karlin, T.W. Anderson et al. (eds), Academic Press, New York, 1989; pp. 27-39. MR.1031276 (91f:33006)

[14] R. Askey and J. Wilson, Some basic hypergeometric orthogonal polynomials that generalize Jacobi polynomials, Mem. Amer. Math. Soc. 54 (1985), Vol. 319. MR783216 (87a:05023)

[15] T.H. Baker and P.J. Forrester, Finite- $N$ fluctuation formulas for random matrices, J. Stat. Phys. 88 (1997), 1371-1386. MR 1478074 (98m:82029)

[16] T.H. Baker and P.J. Forrester, The Calogero-Sutherland model and generalized classical polynomials, Comm. Math. Phys. 188 (1997), 175-216. MR1471336 (99c:33012)

[17] T.H. Baker and P.J. Forrester, Nonsymmetric Jack polynomials and integral kernels, Duke Math. J. 95 (1998), 1-50. MR.1646546 (2000b:33006)

[18] E.W. Barnes, On the theory of the multiple gamma function, Trans. Cambridge Phil. Soc. 19 (1904), 374-425.

[19] D. Barsky and M. Carpentier, Polynômes de Jacobi généralisés et intégrales de Selberg, Electron. J. Combin. 3 (1996), R1. MR1392486 (97k:33005)

[20] R.J. Beerends and E.M. Opdam, Certain hypergeometric series related to the root system $B C$, Trans. Amer. Math. Soc. 339 (1993), 581-609. MR.1123450 (94e:33024)

[21] M.C. Bergère, Proof of Serban's conjecture, J. Math. Phys. 39 (1998), 30-46. MR1489608 (99b:81287)

[22] R.P. Boas, Comments on [132], in George Polya, Collected Papers, Vol. 1, R.P. Boas (ed.), M.I.T. Press, Cambridge, 1974; pp. 771-773. MR0505093 (58:21341)

[23] R.P. Boas Jr., Book Reviews: New Journals Bull. Amer. Math. Soc. 60 (1954), 92-93.

[24] E. Bombieri, Private correspondence, 28 August 2007.

[25] A. Borodin and G. Olshanski, z-Measures on partitions, Robinson-Schensted-Knuth correspondence, and $\beta=2$ random matrix ensembles, in Random matrix models and their applications, P.M. Bleher and A.R. Its (eds), MSRI Publ. Vol. 40, Cambridge University Press, Cambridge, 2001; pp. 71-94. MR:1842783 (2002m:82026)

[26] F. Calogero, Solution of a three-body problem in one dimension, J. Math. Phys. 10 (1969), 2191-2196.

[27] F. Carlson, Sur une Classe de Séries de Taylor, Ph.D. thesis, Uppsala Univ. 1914.

[28] I. Cherednik, A unification of the Knizhnik-Zamolodchikov and Dunkl operators via affine Hecke algebras, Inv. Math. 106 (1991), 411-432. MR.1128220 (93b:17040)

[29] I. Cherednik, Double affine Hecke algebras and Macdonald's conjectures, Ann. of Math. 141 (1995), 191-216. MR1314036 (96m:33010)

[30] I. Cherednik, Double affine Hecke algebras, London Mathematical Society Lecture Note Series, Vol. 319, Cambridge University Press, Cambridge, 2005. MR2133033 (2007e:32012) 
[31] I. Cherednik and V. Ostrik, From double Hecke algebra to Fourier transform, Selecta Math. (N.S.) 9 (2003), 161-249. MR.1993484 (2004f:20011)

[32] P.B. Cohen and A. Regev, Asymptotics of multinomial sums and identities between multiintegrals, Israel J. Math. 112 (1999), 301-325. MR1714982 (2000h:05228)

[33] A.G. Constantine, Some noncentral distribution problems in multivariate analysis, Ann. Math. Statist. 34 (1963), 1270-1285. MR0181056 (31:5285)

[34] H. Coskun and R.A. Gustafson, Well-poised Macdonald functions $W_{\lambda}$ and Jackson coeffcients $\omega_{\lambda}$ on $B C_{n}$, Contemp. Math. 417 (2006), 127-155. MR2284125 (2008f:05197)

[35] A.W. Davis, On the marginal distributions of the latent roots of the multivariate beta matrix, Ann. Math. Stat. 43 (1972), 1664-1669. MR0343465 (49:8206)

[36] A. Debiard, Système différentiel hypergéométrique et parties radiales des opérateurs invariants des espaces symétriques de type $B C_{p}$, Lecture Notes in Math. Vol. 1296, Springer, Berlin, 1987; pp. 42-124. MR932052 (89e:22027)

[37] J.F. van Diejen, Self-dual Koornwinder-Macdonald polynomials, Invent. Math. 126 (1996), 319-339. MR1411136 (98k:33025)

[38] J.F. van Diejen, Confluent hypergeometric orthogonal polynomials related to the rational quantum Calogero system with harmonic confinement, Comm. Math. Phys. 188 (1997), 467-497. MR1471824 (98j:33007)

[39] J.F. van Diejen and V.P. Spiridonov, Elliptic Selberg integrals, Internat. Math. Res. Notices (2001), 1083-1110. MR:1857597 (2002j:33016)

[40] A.L. Dixon, Generalizations of Legendre's formula $K E^{\prime}-(K-E) K^{\prime}=\frac{1}{2} \pi$, Proc. London Math. Soc. 3 (1905), 206-224.

[41] V.S. Dotsenko and V.A. Fateev, Four-point correlation functions and the operator algebra in $2 D$ conformal invariant theories with central charge $C \leq 1$, Nucl. Phys. B 251 (1985), 691-734. MR789026 (86m:81100a)

[42] I. Dumitriu and A. Edelman, Matrix models for beta ensembles, J. Math. Phys. 43 (2002), 5830-5847. MR 1936554 (2004g:82044)

[43] C.F. Dunkl, Differential-difference operators associated to reflection groups, Trans. Amer. Math. Soc. 311 (1989), 167-183. MR951883 (90k:33027)

[44] C.F. Dunkl and Y. Xu, Orthogonal polynomials of several variables, Encyclopedia of Mathematics and its Applications, Vol. 81, Cambridge University Press, Cambridge, 2001. MR:1827871 (2002m:33001)

[45] F.J. Dyson, Statistical theory of energy levels of complex systems. I, J. Math. Phys. 3 (1962), 140-156. MR0143556(26:1111)

[46] P.I. Etingof, I.B. Frenkel and A.A. Kirillov, Jr, Lectures on Representation Theory and Knizhnik-Zamolodchikov Equations, Mathematical Surveys and Monographs, Vol. 58, Amer. Math. Soc., Providence, RI, 2003. MR1629472 (2001b:32028)

[47] L. Euler, De progressionibus transcendentibus seu quarum termini generales algebraice dari nequeunt, Comm. Acad. Sci. Petropolitanae 5 (1730), 36-57.

[48] L. Euler, Institutiones Calculi Integralis, II, Opera Omnia, Ser. 1, Vol. 12.

[49] R.J. Evans, Identities for products of Gauss sums over finite fields, L'Enseignement Math. 27 (1981), 197-209. MR659148(83i:10050)

[50] R.J. Evans, The evaluation of Selberg character sums, L'Enseignement Math. 37 (1991), 235-248. MR1151749 (93c:11062)

[51] R.J. Evans, Multidimensional q-beta integrals, SIAM J. Math. Anal. 23 (1992), 758-765. MR1158832(93e:33004)

[52] R.J. Evans, Multidimensional beta and gamma integrals, Contemp. Math. 166 (1994), 341357. MR 1284073 (96e:33036)

[53] R.J. Evans, Selberg-Jack character sums of dimension 2, J. Number Theory 54 (1995), 1-11. MR:1352632 (96m:11068)

[54] R.J. Evans, Private correspondence, 31 August 2007.

[55] R.J. Evans and W.A. Root, Conjectures for Selberg character sums, J. Ramanujan Math. Soc. 3 (1988), 111-128. MR975841 (90e:11120)

[56] G. Felder, L. Stevens and A. Varchenko, Elliptic Selberg integrals and conformal blocks, Math. Res. Lett. 10 (2003), 671-684. MR2024724(2004i:32024)

[57] P.J. Forrester, Recurrence equations for the computation of correlations in the $1 / r^{2}$ quantum many body system, J. Stat. Phys. 72 (1993), 39-50. MR:1233026 (94e:82009)

[58] P.J. Forrester, Log-Gases and Random Matrices, http://www.ms.unimelb.edu.au/matpjf 
[59] P.J. Forrester, Beta random matrix ensembles, to appear, Proceedings of the IMS (Singapore) programme on Random Matrix Theory and its Applications to Statistics and Wireless Communications.

[60] P.J. Forrester, A random matrix decimation procedure relating $\beta=2 /(r+1)$ to $\beta=2(r+1)$, to appear in Commun. Math. Phys.; arXiv:0711.1914.

[61] P.J. Forrester and E.M. Rains, Interpretations of some parameter dependent generalizations of classical matrix ensembles, Probab. Theory Relat. Fields 131 (2005), 1-61. MR2105043 (2006g:05222)

[62] P.J. Forrester and E.M. Rains, in preparation.

[63] F.G. Garvan, Some Macdonald-Mehta integrals by brute force, in $q$-Series and Partitions, IMA Vol. Math. Appl. 18, Springer, New York, 1989; pp. 77-98. MR1019845 (90k:33030)

[64] F.G. Garvan, Unpublished computer proof of the $k=1$ case of (1.20) for $\mathrm{H}_{4}$.

[65] F.G. Garvan, A proof of the Macdonald-Morris root system conjecture for $F_{4}$, SIAM J. Math. Anal. 21 (1990), 803-821. MR.1046804(91d:33001)

[66] F.G. Garvan and G. Gonnet, Macdonald's constant term conjectures for exceptional root systems, Bull. Amer. Math. Soc. (N.S.) 24 (1991), 343-347. MR.1078471 (92b:33054)

[67] G. Gasper and M. Rahman, Basic Hypergeometric Series, Encyclopedia of Mathematics and its Applications, Vol. 35, second edition, Cambridge University Press, Cambridge, 2004. MR2128719(2006d:33028)

[68] A.O. Gelfond, Sur un theorème de M.G. Polya, Atti Reale Accad. Naz Lincei 10 (1929), $569-574$.

[69] I.J. Good, Short proof of a conjecture of Dyson, J. Math. Phys. 11 (1970), 1884. MR0258644 $(41: 3290)$

[70] J. Gunson, Proof of a conjecture of Dyson in the statistical theory of energy levels, J. Math. Phys. 3 (1962), 752-753. MR0148401 (26:5908)

[71] R.A. Gustafson, A generalization of the Selberg beta-integral, Bull. Am. Math. Soc. 22 (1990), 97-105. MR1001607(90j:33001)

[72] R.A. Gustafson, Some q-beta integrals on $S U(n)$ and $S p(n)$ that generalize the AskeyWilson and Nassrallah-Rahman integrals, SIAM J. Math. Anal. 25 (1994), 441-449. MR.1266569 (95b:33053)

[73] L. Habsieger, La q-conjecture de Macdonald-Morris pour $G_{2}$, C.R. Acad. Sc. Paris Sér I 303 (1986), 211-214. MR860819 (87k:17019)

[74] L. Habsieger, Une q-intégrale de Selberg et Askey, SIAM J. Math. Anal. 19 (1988), 14751489. MR.965268 (89m:33002)

[75] G.H. Hardy and J.E. Littlewood, Contributions to the theory of the Riemann zeta-function and the theory of the distribution of primes, Acta Math. 41 (1918), 119-196. MR.1555148

[76] G.J. Heckman, An elementary approach to the hypergeometric shift operators of Opdam, Invent. Math. 103 (1991), 341-350. MR.1085111 (92i:33012)

[77] C.S. Herz, Bessel functions of matrix argument, Ann. Math. 61 (1955), 474-523. MR0069960 (16:1107e)

[78] L.K. Hua, Harmonic analysis of functions of several complex variables in the classical domains, Translations of Mathematical Monographs, Vol. 6, AMS, Providence, RI, 1979. MR598469 (82c:32032)

[79] Institute for Advanced Study, press releases: Atle Selberg 1917-2007, http:/www.ias.edu/ newsroom/announcements/view/1186683853.html

[80] M.E.H. Ismail, Classical and Quantum Orthogonal Polynomials in One Variable, Encyclopedia of Mathematics and its Applications, Vol. 98, Cambridge University Press, Cambridge, 2005. MR2191786 (2007f:33001)

[81] M. Ito, On a theta product formula for Jackson integrals associated with root systems of rank two, J. Math. Anal. Appl. 216 (1997), 122-163. MR1487257(99e:33023)

[82] K. Johansson, Shape fluctuations and random matrices, Comm. Math. Phys. 209 (2000), 437-476. MR:1737991 (2001h:60177)

[83] K. Johansson, Non-intersecting paths, random tilings and random matrices, Prob. Theory Rel. Fields 123 (2002), 225-280. MR1900323 (2003h:15035)

[84] K.W.J. Kadell, A proof of some q-analogues of Selberg's integral for $k=1$, SIAM J. Math. Analysis 19 (1988), 944-968. MR946654 (89h:33006a)

[85] K.W.J. Kadell, A proof of Askey's conjectured q-analogue of Selberg's integral and a conjecture of Morris, SIAM J. Math. Analysis 19 (1988), 969-986. MR946655 (89h:33006b) 
[86] K.W.J. Kadell, An integral for the product of two Selberg-Jack symmetric polynomials, Compositio Math. 87 (1993), 5-43. MR1219451 (95e:33001)

[87] K.W.J. Kadell, A proof of the $q$-Macdonald-Morris conjecture for $B C_{n}$, Mem. Amer. Math. Soc. 108 (1994), Vol. 516. MR.1140650 (94h:33013)

[88] K.W.J. Kadell, The Selberg-Jack symmetric functions, Adv. Math. 130 (1997), 33-102. MR1467311(98k:05141)

[89] S. Kakei, Intertwining operators for a degenerate double affine Hecke algebra and multivariable orthogonal polynomials, J. Math. Phys. 39 (1998), 4993-5006. MR:1643322 (99h:33049)

[90] J. Kaneko, Selberg integrals and hypergeometric functions associated with Jack polynomials, SIAM J. Math Anal. 24 (1993), 1086-1110. MR1226865(94h:33010)

[91] J. Kaneko, q-Selberg integrals and Macdonald polynomials, Ann. Sci. École Norm. Sup. (4) 29 (1996), 583-637. MR1399617 (98k:33026)

[92] S. Karlin and L.S. Shapley, Geometry of moment space, Mem. Amer. Math. Soc. (1953), Vol. 12. MR0059329 (15:512c)

[93] N. Katz and P. Sarnak, Zeroes of zeta functions and symmetry, Bull. Amer. Math. Soc. 36 (1999), 1-26. MR1640151 (2000f:11114)

[94] J.P. Keating and N.C. Snaith, Random matrix theory and $\zeta(1 / 2+i t)$, Comm. Math. Phys. 214 (2001), 57-89. MR 1794265 (2002c:11107)

[95] J.P. Keating and N.C. Snaith, Random matrix theory and L-functions at $s=1 / 2$, Comm. Math. Phys. 214 (2001), 91-110. MR1794267 (2002c:11108)

[96] J.P. Keating and N.C. Snaith, Random matrices and L-functions, J. Phys. A 36 (2003), 2859-2882. MR.1986396 (2004d:11090)

[97] J.P. Keating, N. Linden and Z. Rudnick, Random matrix theory, the exceptional Lie groups and L-functions, J. Phys. A 36 (2003), 2933-2944. MR1986400 (2004f:11094)

[98] R. Killip and I. Nenciu, Matrix models for circular ensembles, Int. Math. Res. Not. 50 (2004), 2665-2701. MR2127367 (2006h:82003)

[99] V.G. Knizhnik and A.B. Zamolodchikov, Current algebra and Wess-Zumino model in two dimensions, Nuclear Phys. B 247 (1984), 83-103. MR853258 (87h:81129)

[100] T.H. Koornwinder, Askey-Wilson polynomials for root systems of type BC, Contemp. Math. 138 (1992), 189-204. MR.1199128 (94e:33039)

[101] A. Korányi, Hua-type integrals, hypergeometric functions and symmetric polynomials, in International Symposium in Memory of Hua Loo Keng, Vol. II, Springer, Berlin, 1991; pp. 169-180. MR.1135834 (92h:33036)

[102] M. Lassalle, Polynômes de Jacobi généralisés, C. R. Acad. Sci. Paris Sér. I Math. 312 (1991), 425-428. MR1096625 (92g:33019)

[103] M. Lassalle, Polynômes de Laguerre généralisés, C. R. Acad. Sci. Paris Sér. I Math. 312 (1991), 725-728. MR1105634 (92g:33018)

[104] M. Lassalle, Polynômes de Hermite généralisés, C. R. Acad. Sci. Paris Sér. I Math. 313 (1991), 579-582. MR1133488 (93a:33020)

[105] J.-G. Luque and J.-Y. Thibon, Hankel hyperdeterminants and Selberg integrals, J. Phys. A 36 (2003), 5267-5292. MR1985318 (2004d:15011)

[106] J.-G. Luque and J.-Y. Thibon, Hyperdeterminantal calculations of Selberg's and Aomoto's integrals, Molecular Physics 102 (2004), 1351-1359.

[107] B. Nassrallah and M. Rahman, Projection formulas, a reproducing kernel and a generating function for $q$-Wilson polynomials, SIAM J. Math. Anal. 16 (1985), 186-197. MR772878 (87b:33009)

[108] I.G. Macdonald, Some conjectures for root systems, SIAM J. Math. Anal. 13 (1982), 9881007. MR674768 (84h:17006a)

[109] I.G. Macdonald, Commuting differential operators and zonal spherical functions, Lecture Notes in Math. 1271 (1987), 189-200. MR911140 (89e:43025)

[110] I.G. Macdonald, Hall polynomials and symmetric functions, 2nd ed., Oxford University Press, Oxford, 1995. MR1354144 (96h:05207)

[111] I.G. Macdonald, Affine Hecke algebras and orthogonal polynomials, Cambridge Tracts in Mathematics, Vol. 157, Cambridge University Press, Cambridge, 2003. MR 1976581 (2005b:33021)

[112] I. G. Macdonald, Hypergeometric functions, unpublished manuscript.

[113] M.L. Mehta, Random Matrices and the Statistical Theory of Energy Levels, Academic Press, New York, 1967. MR0220494 (36:3554) 
[114] M.L. Mehta, Problem 74-6, Three multiple integrals, SIAM Review 16 (1974), 256-257.

[115] M.L. Mehta, Random Matrices, 3rd ed., Pure and Applied Mathematics, Vol. 142, Elsevier/Academic Press, Amsterdam, 2004. MR2129906 (2006b:82001)

[116] M.L. Mehta and F.J. Dyson, Statistical theory of the energy levels of complex systems. $V$, J. Math. Phys. 4 (1963), 713-719. MR0151232 (27:1218)

[117] K. Mimachi, Reducibility and irreducibility of the Gauss-Manin system associated with a Selberg type integral, 132 (1993), 43-62. MR.1253694 (95a:33034)

[118] K. Mimachi, The connection problem associated with a Selberg type integral and the $q$-Racah polynomials, arXiv:0710.2167.

[119] K. Mimachi and M. Yoshida, The reciprocity relation of the Selberg function, in Proceedings of the International Conference on Special Functions and their Applications (Chennai, 2002), J. Comput. Appl. Math. 160 (2003), 209-215. MR2022612(2004k:33003)

[120] K. Mimachi and M. Yoshida, Intersection numbers of twisted cycles associated with the Selberg integral and an application to the conformal field theory, Comm. Math. Phys. 250 (2004), 23-45. MR2092027 (2006c:32034)

[121] K. Mimachi and T. Takamuki, A generalization of the beta integral arising from the Knizhnik-Zamolodchikov equation for the vector representations of types $B_{n}, C_{n}$ and $D_{n}$, Kyushu J. Math. 59 (2005), 117-126. MR2134056 (2006m:33001)

[122] W.G. Morris, Constant Term Identities for Finite and Affine Root Systems: Conjectures and Theorems, Ph.D. thesis, Univ. Wisconsin-Madison, 1982.

[123] R. J. Muirhead, Systems of partial differential equations for hypergeometric functions of matrix argument, Ann. Math. Statist. 41 (1970), 991-1001. MR0264799 (41:9390)

[124] E. Mukhin and A. Varchenko, Remarks on critical points of phase functions and norms of Bethe vectors, Adv. Stud. Pure Math. 27 (2000), 239-246. MR.1796902 (2001j:32012)

[125] A.M. Odlyzko, The $10^{20}$ th zero of the Riemann zeta function and 70 million of its neighbours, http://www.dtc.umn.edu/ odlyzko/unpublished/index.html

[126] A. Okounkov, (Shifted) Macdonald polynomials: q-integral representation and combinatorial formula, Compositio Math. 112 (1998), 147-182. MR.1626029 (99h:05120)

[127] A. Okounkov, BC-type interpolation Macdonald polynomials and binomial formula for Koornwinder polynomials, Transform. Groups 3 (1998) 181-207. MR1628453 (99h:33061)

[128] A. Okounkov, Infinite wedge and random partitions, Selecta Math., New Ser. 7 (2001), 57-81. MR.1856553 (2002f:60019)

[129] E.M. Opdam, Some applications of hypergeometric shift operators, Invent. Math. 98 (1989), 1-18. MR:1010152 (91h:33024)

[130] E.M. Opdam, Dunkl operators, Bessel functions and the discriminant of a finite Coxeter group, Compositio Math. 85 (1993), 333-373. MR1214452 (95j:33044)

[131] E.M. Opdam, Lecture notes on Dunkl operators for real and complex reflection groups, MSJ Memoirs, Vol. 8, Math. Soc. Japan, Tokyo, 2000. MR1805058(2003c:33001)

[132] G. Polya, Collected Papers, Vol. 1, R.P. Boas (ed.), M.I.T. Press, Cambridge, 1974; pp. 116. MR0505093 (58:21341)

[133] M. Rahman, An integral representation of a ${ }_{10} \varphi_{9}$ and continuous bi-orthogonal ${ }_{10} \varphi_{9}$ rational functions, Canad. J. Math. 38 (1986), 605-618. MR845667(87i:33011)

[134] E.M. Rains, Transformations of elliptic hypergeometric integrals, to appear in Ann. of Math.; arXiv:math.QA/0309252.

[135] E.M. Rains, Private communication.

[136] E.M. Rains, Limits of elliptic hypergeometric integrals, to appear in Ramanujan J.; arXiv:math.CA/0607093.

[137] E.M. Rains and V.P. Spiridonov, Determinants of elliptic hypergeometric integrals, to appear in Funct. Anal. Appl.; arXiv:0712.4253

[138] A. Regev, Asymptotic values for degrees associated with strips of Young diagrams, Adv. in Math. 41 (1981), 115-136. MR625890 (82h:20015)

[139] A. Regev, Private correspondence, 30 August 2007.

[140] D. Richards and Q. Zheng, Determinants of period matrices and an application to Selberg's multidimensional beta integral, Adv. in Appl. Math. 28 (2002), 602-633. MR.1900011 (2003f:33018) 
[141] M. Rubinstein, Computational methods and experiments in analytic number theory, in Recent Perspectives in Random Matrix Theory and Number Theory, F. Mezzadri and N.C. Snaith (eds), LMS Lecture Note Series 322, Cambridge University Press, Cambridge, 2005; pp. 425-506. MR2166470 (2006d:11153)

[142] S.N.M. Ruijsenaars, First order analytic difference equations and integrable quantum systems, J. Math. Phys. 38 (1997), 1069-1146. MR1434226 (98m:58065)

[143] V.V. Schechtman and A.N. Varchenko, Arrangements of hyperplanes and Lie algebra homology, Invent. Math. 106 (1991), 139-194. MR1123378 (93b:17067)

[144] A. Selberg, Über einen Satz von A. Gelfond, Arch. Math. Naturvid. 44 (1941) 159-171. MR0006569 (4:6f)

[145] A. Selberg, Bemerkninger om et multipelt integral, Norsk. Mat. Tidsskr. 24 (1944), 71-78. MR0018287 (8:269b)

[146] A. Selberg, Contributions to the theory of the Riemann zeta-function, Arch. Math. OG. Naturv. B 48 (1946), 89-155. (Reprinted with commentary in [147].) MR0020594 (8:567e)

[147] A. Selberg, Collected papers I, Springer-Verlag, Heidelberg, 1989; p. 212. MR.1117906 (92h:01083)

[148] V.P. Spiridonov, On the elliptic beta function, Russ. Math. Surveys 56 (2001), 185-186. MR.1846786 (2003b:33032)

[149] V.P. Spiridonov, Elliptic beta integrals and special functions of hypergeometric type, in Integrable structures of exactly solvable two-dimensional models of quantum field theory, NATO Sci. Ser. II Math. Phys. Chem. Vol. 35, Kluwer Acad. Publ., Dordrecht; pp. 305313. MR.1873579 (2003b:33019)

[150] V.P. Spiridonov, Short proofs of the elliptic beta integrals, Ramanujan J. 13 (2007), 265-283. MR2281166 (2007i:33042)

[151] V.P. Spiridonov, Elliptic hypergeometric functions, A complement to [6], written for its Russian edition; arXiv:0704.3099.

[152] R.P. Stanley, Some combinatorial properties of Jack symmetric functions, Adv. Math. 77 (1989), 76-115. MR1014073 (90g:05020)

[153] R.P. Stanley, Enumerative Combinatorics, Vol. I, Cambridge University Press, New York/Cambridge, 1997. MR.1442260 (98a:05001)

[154] R.P. Stanley, Queue problems revisited, Suomen Tehtäväniekat 59 (2005), 193-203.

[155] R.P. Stanley, Private correspondence, 15 September 2007.

[156] J.R. Stembridge, A short proof of Macdonald's conjecture for the root systems of type A, Proc. Amer. Math. Soc. 102 (1988), 777-786. MR934842 (89e:11062)

[157] J.V. Stokman, Multivariable big and little q-Jacobi polynomials, SIAM J. Math. Anal. 28 (1997), 452-480. MR1434045 (98f:33034)

[158] J.V. Stokman, On BC type basic hypergeometric orthogonal polynomials, Trans. Amer. Math. Soc. 352 (2000), 1527-1579. MR1694379 (2001j:33015)

[159] B. Sutherland, Exact results for a quantum many body problem in one-dimension, Phys. Rev. A 4 (1971), 2019-2021.

[160] B. Sutherland, Exact results for a quantum many-body problem in one dimension: II, Phys. Rev. A 5 (1972), 1372-1376.

[161] V. Tarasov and A. Varchenko, Difference equations compatible with trigonometric KZ differential equations, Internat. Math. Res. Notices 2000, 801-829. MR1780748(2001k:32025)

[162] V. Tarasov and A. Varchenko, Selberg-type integrals associated with $\mathfrak{s l}_{3}$, Lett. Math. Phys. 65 (2003), 173-185. MR2033704 (2004k:33027)

[163] A. Varchenko, The Euler beta-function, the Vandermonde determinant, the Legendre equation, and critical values of linear functions on a configuration of hyperplanes. I, Math. USSR 35 (1990), 543-571. MR1039962 (91c:32031a)

[164] A. Varchenko, The Euler beta-function, the Vandermonde determinant, the Legendre equation, and critical values of linear functions on a configuration of hyperplanes. II, Math. USSR 36 (1991), 155-167. MR1044052 (91c:32031b)

[165] A. Varchenko, Special Functions, KZ Type Equations, and Representation Theory, CBMS Regional Conference Series in Mathematics, Vol. 98, Amer. Math. Soc., Providence, RI, 2003. MR2016311 (2005g:33021)

[166] S.O. Warnaar, q-Selberg integrals and Macdonald polynomials, Ramanujan J. 10 (2005), 237-268. MR2195565 (2007e:33014) 
[167] S.O. Warnaar, On the generalised Selberg integral of Richards and Zheng, Adv. Appl. Math. 40 (2008), 212-218. MR 2388612

[168] S.O. Warnaar, Bisymmetric functions, Macdonald polynomials and $\mathfrak{s l}_{3}$ basic hypergeometric series, Compositio Math. 114 (2008), 271-303.

[169] S.O. Warnaar, A Selberg integral for the Lie algebra $A_{n}$, to appear in Acta Math.; arXiv:0708.1193.

[170] S.O. Warnaar, The Mukhin-Varchenko conjecture for type A, to appear in the Proceedings of FPSAC 2008, Discrete Math. Theor. Comput. Sci.

[171] K. Wilson, Proof of a conjecture of Dyson, J. Math. Phys. 3 (1962), 1040-1043. MR0144627 $(26: 2170)$

[172] E.T. Whittaker and G.N. Watson, A course of modern analysis, Reprint of the fourth (1927) edition, Cambridge University Press, Cambridge, 1996. MR 1424469 (97k:01072)

[173] Z. Yan, A class of generalized hypergeometric functions in several variables, Can J. Math. 44 (1992), 1317-1338. MR1192421 (94c:33026)

[174] D. Zeilberger and D.M. Bressoud, A proof of Andrews' q-Dyson conjecture, Discrete Math. 54 (1985), 201-224. MR791661 (87f:05015)

[175] D. Zeilberger, A proof of the $G_{2}$ case of Macdonald's root system-Dyson conjecture, SIAM J. Math. Anal. 18 (1987), 880-883. MR883574 (88f:05017)

[176] D. Zeilberger, A unified approach to Macdonald's root system conjecture, SIAM J. Math. Anal. 19 (1988), 987-1013. MR946656 (90b:17015)

[177] D. Zeilberger, A Stembridge-Stanton style proof of the Habsieger-Kadell q-Morris identity, Discrete Math. 79 (1989), 313-322. MR1044230(91b:05024)

Department of Mathematics and Statistics, The University of Melbourne, Victoria 3010, Australia

Department of Mathematics, The University of Queensland, St Lucia, Queensland 4072, Australia 